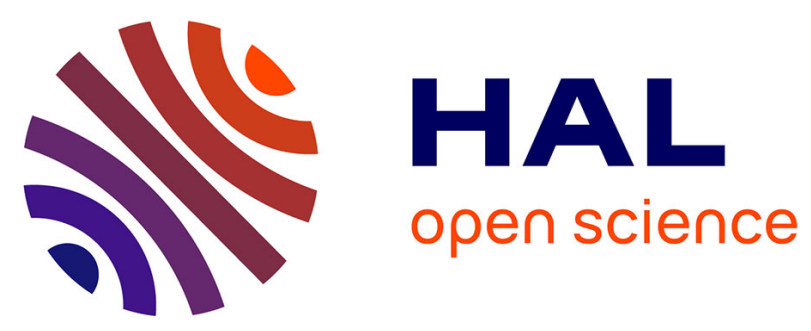

\title{
General coupling of porous flows and hyperelastic formulations - From thermodynamics principles to energy balance and compatible time schemes
}

\author{
Dominique Chapelle, Philippe Moireau
}

\section{- To cite this version:}

Dominique Chapelle, Philippe Moireau. General coupling of porous flows and hyperelastic formulations - From thermodynamics principles to energy balance and compatible time schemes. European Journal of Mechanics - B/Fluids, 2014, 46, pp.82-96. 10.1016/j.euromechflu.2014.02.009 . inria$00520612 \mathrm{v} 3$

\section{HAL Id: inria-00520612 \\ https://hal.inria.fr/inria-00520612v3}

Submitted on 18 Feb 2014

HAL is a multi-disciplinary open access archive for the deposit and dissemination of scientific research documents, whether they are published or not. The documents may come from teaching and research institutions in France or abroad, or from public or private research centers.
L'archive ouverte pluridisciplinaire HAL, est destinée au dépôt et à la diffusion de documents scientifiques de niveau recherche, publiés ou non, émanant des établissements d'enseignement et de recherche français ou étrangers, des laboratoires publics ou privés. 


\title{
General coupling of porous flows and hyperelastic formulations From thermodynamics principles to energy balance and compatible time schemes
}

\author{
D. Chapelle and P. Moireau \\ Inria Saclay Ile-de-France, 1 rue Honoré d'Estienne d'Orves, 91120 Palaiseau, France \\ European Journal of Mechanics / B Fluids, DOI:10.1016/j.euromechflu.2014.02.009
}

\begin{abstract}
We formulate a general poromechanics model - within the framework of a two-phase mixture theory - compatible with large strains and without any simplification in the momentum expressions, in particular concerning the fluid flows. The only specific assumptions made are fluid incompressibility and isothermal conditions. Our formulation is based on fundamental physical principles - namely, essential conservation and thermodynamics laws - and we thus obtain a Clausius-Duhem inequality which is crucial for devising compatible constitutive laws. We then propose to model the solid behavior based on a generalized hyperelastic free energy potential - with additional viscous effects - which allows to represent a wide range of mechanical behaviors. The resulting formulation takes the form of a coupled system similar to a fluid-structure interaction problem written in an Arbitrary Lagrangian-Eulerian formalism, with additional volume-distributed interaction forces. We achieve another important objective by identifying the essential energy balance prevailing in the model, and this paves the way for further works on mathematical analyses, and time and space discretizations of the formulation.
\end{abstract}

Keywords: poromechanics; mixtures; hyperelasticity; thermomechanics; thermodynamics; biomechanics

\section{Introduction}

Poromechanics has been a very active subject of research for some decades - as shown e.g. in the survey [17] - originally mostly motivated by civil engineering applications, see [5, 47], and well-established theories rigorously grounded in fundamental physical and thermodynamical principles are available $[11,16,38,9]$. Such approaches are frequently based on the so-called "mixture theory", a purely macroscopic framework in which the porous medium is considered to be made of a superposition of solid and fluid phases at each point - for a saturated medium to which we restrict our attention. We note in passing that the detailed microstructure can be considered in alternative approaches, e.g. with homogenization procedures applied to relate the microscopic and macroscopic behaviors [30, 41], but we do not dwell on these approaches which are much more difficult to translate into effective computational modeling tools.

More recently, some novel applications have brought renewed modeling and computing challenges in the field, hence spurred further research to circumvent various limitations of the existing theories. In particular, potential challenging applications abound in biomechanics, for instance to model the blood perfusion of living (passive or active) tissues [31, 39, 45], the circulation of gases in the lungs [46,34,3], or the effect of wind on plants [18], to cite just a few noteworthy examples in this emerging context. 
In this context, in [13] a poromechanics formulation was proposed with the primary motivation of describing perfusion in the cardiac muscle. As the heart typically undergoes strains of 10 to $20 \%$, the focus of this work was accordingly placed on the ability of the model to represent large displacements and finite strains, with general constitutive laws compatible with living tissue behavior $[25,29]$. In addition, special care was exercised to make the proposed formulation consistent with - partial or complete - incompressibility of the solid and fluid constituents. However, a significant - although quite widespread - simplification was made in this work by totally neglecting the fluid inertia. As the coronary arteries are known to be host to rapid blood flows with high variations during the cardiac cycle - peak flow velocity being typically of 10-20 $\mathrm{cm} . \mathrm{s}^{-1}$ [33], i.e. similar to peak solid velocity, albeit occurring at different, alternating, times - such a simplification is clearly quite drastic, and furthermore does not allow the formulation of discretization procedures which would preserve physical energy balances - with the kinetic energy as one of the major contributions.

The general principles of poromechanics formulations fulfilling fundamental conservation and thermodynamics laws have long been established, see in particular $[11,44,12]$ and references therein. However, even in a rather general framework the development of effective formulations need also take into account the specificities and constraints of the category of applications considered, and for example in the above-cited pioneering works fluid inertia is rapidly discarded, and the issue of deriving general constitutive laws based on existing complex hyperelastic potentials is not addressed. By contrast, some other poromechanics formulations taking into account finite strains - and with or without fluid inertia - have already been proposed, see e.g. [19, 1, 37]. However, these formulations are mostly directly focused on constitutive assumptions - and indeed pertain to specific types of constitutive behaviors - rather than explicitly derived from the general setting of conservation and thermodynamics principles. Hence, it is difficult to see whether these essential principles are satisfied, and how more general constitutive behaviors can be adequately modeled within the proper corresponding physical framework, in particular as regards energy considerations.

The objective of the present work is thus twofold. We first aim at presenting a general poromechanics theory compatible with large strains and porous fluid flows without any simplification in the momentum expressions, based on fundamental physical principles - namely, essential conservation and thermodynamics laws. In this setting, arbitrary hyperelastic potentials can be used to represent the skeleton behavior, and the transition between compressible and incompressible behaviors - a key distinction in many existing poromechanics theories - is here unified in a seamless manner. This construction based on fundamental principles allows us to meet a second objective, namely, to identify the essential energy balance which must be satisfied in such formulations. This is crucial in order to allow further mathematical analyses and the derivation of consistently stable time and space discretization procedures. Our motivation is - indeed - strongly oriented towards computational modeling. As an example, we demonstrate the derivation of a compatible time scheme, as the first important step in the numerical simulation workflow. We note in passing that - when applied to cardiac perfusion - this formulation should allow to comparatively assess the impact of the simplifications made in earlier works.

We should mention that our approach is clearly inspired from the presentation of [16] - see also [21] and references therein - itself largely based on the pioneering theory of Biot [6, 7], in particular as regards the concept of mixture considered in a thermodynamics setting and the Lagrangian formalism attached to the solid phase. In fact, we essentially follow the same major construction steps as in [16], and we herein summarize these steps both for completeness and in order to emphasize our specific distinctions and extensions. Namely, our main contributions thus lie in: 
- the detailed generalization of this framework to nonlinear constitutive behaviors modeled by hyperelastic potentials and viscous effects - both in the solid and in the fluid - with a proposed systematic construction method for introducing these features based on general modeling ingredients already available from solid and fluid mechanics separately, and with a particular concern for incompressible or nearly-incompressible behaviors on both sides;

- the incorporation of fluid mass source terms in the formulation, these being motivated in particular by the modeling of muscle tissue in its interaction with various blood compartments providing input and output distributed coupling conditions [13];

- establishing the relation between the resulting coupled variational formulation, and socalled "Arbitrary Lagrangian-Eulerian" (ALE) formulations of fluid-structure interaction problems [20], which paves the way for further analyses and numerical considerations, as exemplified in a proposed time discretization scheme.

The outline of the paper is as follows. In Section 2 we introduce the notation and kinematical description, and we derive the mass conservation laws. We then establish in Section 3 the adequate principle of virtual work by invoking the conservation of momentum. In Section 4 we obtain the energy conservation law based on the first fundamental principle of thermodynamics, before proceeding to apply the second principle in Section 5, which - combined with the previous results - leads to a Clausius-Duhem inequality allowing to formulate consistent constitutive laws. Next, in Section 6 we summarize and further analyze the governing equations of the complete model - both in strong and weak forms - and we establish a fundamental energy balance. This leads us to providing an example of consistent time scheme in Section 7, before giving some concluding remarks in Section 8.

\section{Notation, kinematical description and mass conservation}

In this section we start by introducing the notation and kinematical description, and proceed to derive the mass conservation laws.

\subsection{Classical definitions and kinematical relations}

We consider a deformable solid which occupies at time $t$ the space domain $\Omega(t)$ - denoted by $\Omega$ when there is no ambiguity- with boundary $\partial \Omega(t)$. The total Lagrangian formulation consists in describing the position history of each material point with respect to a reference configuration $\left(\Omega_{0}, \partial \Omega_{0}\right)$ - not necessarily equal to $(\Omega(0), \partial \Omega(0))$. Thus, the deformation is a one-to-one mapping $\underline{\varphi}$ from the reference configuration to the current configuration providing the position of each material point in time:

$$
\underline{\varphi}:\left\{\begin{aligned}
\bar{\Omega}_{0} & \rightarrow \mathbb{R}^{3} \\
\underline{\xi} & \mapsto \underline{x}=\underline{\varphi}(\underline{\xi}, t)
\end{aligned}\right.
$$

We denote by $\underline{y}$ the displacement field

$$
\underline{y}(\underline{\xi}, t)=\underline{\mathrm{x}}-\underline{\xi}=\underline{\varphi}(\underline{\xi}, t)-\underline{\xi},
$$

and $\underline{\underline{F}}$ is the deformation gradient

$$
\underline{\underline{F}}(\underline{\xi}, t)=\underline{\underline{\nabla}} \underline{\underline{\varphi}}=\underline{\underline{\mathbb{1}}}+\underline{\underline{\nabla}} \underline{\xi} \underline{y}
$$


such that the deformed volume is given by $J d \Omega$ where $J=\operatorname{det} \underline{\underline{F}}$ and $d \Omega$ is the volume measure (here in the reference configuration), while the deformed area vector is given by $J \underline{\underline{F}}^{-T} \cdot \underline{d S}$. Furthermore, we introduce the right Cauchy-Green deformation tensor $\underline{\underline{C}}=\underline{\underline{F}}^{T} \cdot \underline{\underline{F}}$. We finally recall that the local changes of geometry are conveniently described by the Green-Lagrange strain tensor denoted by $\underline{\underline{e}}$ and defined by

$$
\underline{\underline{e}}=\frac{1}{2}(\underline{\underline{C}}-\underline{\underline{\mathbb{1}}})=\frac{1}{2}\left(\underline{\underline{\nabla}} \underline{\underline{y}}+(\underline{\underline{\nabla}} \underline{\underline{y}})^{T}+(\underline{\underline{\nabla}} \underline{\underline{y}})^{T} \cdot \underline{\underline{\nabla}}_{\underline{\xi}} \underline{y}\right)
$$

with linearized expression $\underline{\underline{\varepsilon}}$ given by

$$
\underline{\underline{\varepsilon}}(\underline{y})=\frac{1}{2}\left(\underline{\underline{\nabla}} \underline{\underline{y}}+(\underline{\underline{\underline{\nabla}}} \underline{\underline{y}})^{T}\right)
$$

\subsection{Mixture and material derivatives}

Classical two-phase poromechanics is a mixture theory in which the material contains at each point a volume fraction $\phi$ of fluid phase, and a remaining fraction $(1-\phi)$ of solid phase called the "skeleton". The fluid volume fraction $\phi$ is also referred to as the "porosity". Unless otherwise specified, we henceforth denote the quantities specifically associated with the fluid and the skeleton with "f" and "s" subscripts, respectively. As an exception, we will retain all the notation introduced in the above Section 2.1 to denote all kinematical quantities associated with the skeleton - without the "s" subscript, since this also describes the motion of the fluid domain. Note that $J$ then only represents an apparent change of volume of the skeleton, since it can be accompanied by a variation of the porosity.

For a field $u_{\mathrm{s}}(\underline{\mathrm{x}}, t)$ associated with the solid skeleton, we recall the classical concept of material derivative defined by

$$
\frac{\mathrm{d} u_{\mathrm{s}}}{\mathrm{dt}}=\left.\frac{\partial\left(u_{\mathrm{s}} \circ \underline{\varphi}\right)}{\partial t}\right|_{\underline{\xi}}=\left.\frac{\partial u_{\mathrm{s}}}{\partial t}\right|_{\underline{\mathrm{x}}}+\underline{\nabla}_{\underline{\mathrm{x}}} u_{\mathrm{s}} \cdot \underline{v}_{\mathrm{s}},
$$

where the velocity $\underline{v}_{\mathrm{s}}$ is defined by

$$
\underline{v}_{\mathrm{s}}=\left.\frac{\partial \underline{\mathrm{x}}}{\partial t}\right|_{\underline{\xi}}
$$

Hence, when $u_{\mathrm{s}}(\underline{\mathrm{x}}, t)$ denotes the volume density associated with an extensive quantity $\mathcal{U}=$ $\int_{\Omega_{t}^{\prime}} u_{\mathrm{S}}(\underline{\mathrm{x}}, t) d \Omega$ defined on the solid skeleton in a Eulerian framework - for an arbitrary subdomain $\Omega_{t}^{\prime} \subset \Omega_{t}$ associated with $\Omega_{0}^{\prime} \subset \Omega_{0}$ in the reference configuration - the material time derivative of the integrated density gives

$$
\begin{aligned}
\frac{\mathrm{d}}{\mathrm{dt}} \int_{\Omega_{t}^{\prime}} u_{\mathrm{s}} d \Omega & =\frac{\mathrm{d}}{\mathrm{dt}} \int_{\Omega_{0}^{\prime}} u_{\mathrm{s}}(\underline{\mathrm{x}}(\underline{\xi}, t), t) J(\underline{\xi}, t) d \Omega \\
& =\int_{\Omega_{0}^{\prime}}\left(\left.J \frac{\partial u_{\mathrm{s}}}{\partial t}\right|_{\underline{\mathbf{x}}}+J \underline{\nabla}_{\underline{\mathrm{x}}} u_{\mathrm{s}} \cdot \underline{v}_{\mathrm{s}}+\left.u_{\mathrm{s}} \frac{\partial J}{\partial t}\right|_{\underline{\xi}}\right) d \Omega .
\end{aligned}
$$

Using the identity

$$
\frac{\partial J}{\partial t}=\frac{\partial}{\partial t} \operatorname{det} \underline{\underline{F}}=\operatorname{tr}\left(J \underline{\underline{F}}^{-1} \cdot \frac{\partial \underline{\underline{F}}}{\partial t}\right)=J \operatorname{tr}\left(\underline{\underline{F}}^{-1} \cdot \underline{\underline{\nabla}}_{\underline{\underline{v}}}\right)=J \operatorname{tr}\left(\underline{\underline{\nabla}}_{\underline{\mathrm{x}}} \underline{\underline{\mathrm{v}}}\right)=J \underline{\underline{\nabla}}_{\underline{\mathrm{x}}} \cdot \underline{v}_{\mathrm{s}},
$$

we get

$$
\frac{\mathrm{d}}{\mathrm{dt}} \int_{\Omega_{t}^{\prime}} u_{\mathrm{S}} d \Omega=\int_{\Omega_{t}^{\prime}}\left(\left.\frac{\partial u_{\mathrm{s}}}{\partial t}\right|_{\underline{\mathbf{x}}}+\underline{\nabla}_{\underline{\mathbf{x}}} \cdot\left(u_{\mathrm{S}} \otimes \underline{v}_{\mathrm{S}}\right)\right) d \Omega,
$$


where we employ a slight abuse of notation by which $u_{\mathrm{s}}$ can denote a tensor of arbitrary order, hence, in case $u_{\mathrm{s}}$ is a scalar then tensor product " $\otimes$ " reduces to standard multiplication.

We can also introduce material derivatives for quantities associated with the fluid

$$
\frac{\mathrm{d}_{\mathrm{f}} u_{\mathrm{f}}}{\mathrm{dt}}=\left.\frac{\partial u_{\mathrm{f}}}{\partial t}\right|_{\underline{\mathrm{x}}}+\underline{\nabla}_{\underline{\mathrm{x}}} u_{\mathrm{f}} \cdot \underline{v}_{\mathrm{f}} .
$$

Remark 1 - We point out that we could of course also introduce a Lagrangian formalism for the fluid giving when differentiating an integral quantity on $\Omega_{t}^{\prime}$

$$
\frac{\mathrm{d}_{\mathrm{f}}}{\mathrm{dt}} \int_{\Omega_{t}^{\prime}} u_{\mathrm{f}} d \Omega=\int_{\Omega_{t}^{\prime}}\left(\left.\frac{\partial u_{\mathrm{f}}}{\partial t}\right|_{\underline{\mathbf{x}}}+\underline{\nabla}_{\underline{\mathrm{x}}} \cdot\left(u_{\mathrm{f}} \otimes \underline{v}_{\mathrm{f}}\right)\right) d \Omega .
$$

Let us now consider an integral quantity $\mathcal{U}=\int_{\Omega_{t}^{\prime}} u(\underline{\mathrm{x}}, t) d \Omega$ defined as the sum of two terms

$$
\mathcal{U}=\int_{\Omega_{t}^{\prime}}\left(u_{\mathrm{s}}+u_{\mathrm{f}}\right) d \Omega
$$

As above, the first term $u_{\mathrm{s}}$ is associated with the solid skeleton, i.e. its velocity is that of the skeleton, namely, $\underline{v}_{\mathrm{s}}$. The second term follows the fluid with the velocity $\underline{v}_{\mathrm{f}}$.

\section{Definition 1 (Total time derivative of a mixture quantity)}

We define the total time derivative of $\mathcal{U}$ as

$$
\frac{\mathrm{D} \mathcal{U}}{\mathrm{Dt}}=\frac{\mathrm{d}}{\mathrm{dt}} \int_{\Omega_{t}^{\prime}} u_{\mathrm{s}} d \Omega+\frac{\mathrm{d}_{\mathrm{f}}}{\mathrm{dt}} \int_{\Omega_{t}^{\prime}} u_{\mathrm{f}} d \Omega .
$$

Introducing $\underline{w}$ the mass weighted relative velocity of the fluid defined by

$$
\underline{w}=\rho_{\mathrm{f}} \phi\left(\underline{v}_{\mathrm{f}}-\underline{v}_{\mathrm{s}}\right)=\rho_{\mathrm{f}} \phi \underline{v}_{\mathrm{r}},
$$

and $u_{m}$ the proportion of $u_{\mathrm{f}}$ per unit fluid mass

$$
u_{m}=\frac{u_{\mathrm{f}}}{\rho_{\mathrm{f}} \phi},
$$

we then have the following rule of differentiation, directly inferred from (2) and (4).

\section{Lemma 2 (Differentiation lemma)}

For a tensor $u$ of arbitrary order, we have

$$
\frac{\mathrm{D} \mathcal{U}}{\mathrm{Dt}}=\int_{\Omega_{t}^{\prime}}\left(\frac{\partial u}{\partial t}+\underline{\nabla}_{\underline{\mathrm{x}}} \cdot\left(u \otimes \underline{v}_{\mathrm{s}}\right)+\underline{\nabla}_{\underline{\mathrm{x}}} \cdot\left(u_{m} \otimes \underline{w}\right)\right) d \Omega .
$$

These relations have equivalent forms when considering Lagrangian coordinates attached to the skeleton and the corresponding derivatives. Defining

$$
U(\underline{\xi}, t)=u(\underline{\mathrm{x}}(\underline{\xi}, t)) J(\underline{\xi}, t)
$$

as the Lagrangian volume density, the first two terms of (5) correspond to the classical partial time derivative of a function defined in the skeleton reference configuration. Therefore, defining $\underline{W}$ as

$$
\underline{W}=J \underline{\underline{F}}^{-1} \underline{w}
$$

such that for any infinitesimal area $\underline{d S_{0}}$ transported into $\underline{d S}$ we have

$$
\underline{W} \cdot \underline{d S}_{0}=\underline{w} \cdot \underline{d S},
$$


the Stokes formula gives

$$
\int_{\Omega_{0}^{\prime}} \underline{\nabla}_{\underline{\xi}} \cdot\left(u_{m} \otimes \underline{W}\right) d \Omega=\int_{\Omega_{t}^{\prime}} \underline{\nabla}_{\underline{\mathbf{x}}} \cdot\left(u_{m} \otimes \underline{w}\right) d \Omega .
$$

This directly implies

$$
\frac{\mathrm{DU}}{\mathrm{Dt}}=\int_{\Omega_{0}}\left(\frac{\partial U}{\partial t}+\underline{\nabla_{\underline{\xi}}} \cdot\left(u_{m} \otimes \underline{W}\right)\right) d \Omega .
$$

Note that in the above formulae we use the same notation for a function of $\underline{\xi}$ and of $\underline{x}$-in this case for $u_{m}$. We will systematically commit this slight abuse of notation for all fields in the sequel.

\subsection{Mass conservation laws}

Lemma 2 can be used to obtain mass conservation laws for the entire system or for the fluid subsystem alone. Starting with the fluid, we consider a distributed mass source term $\theta$ - which represents a source per se if $\theta \geq 0$ and a sink otherwise [13]. We have for any subdomain $\Omega_{t}^{\prime}$

$$
\frac{\mathrm{D}}{\mathrm{Dt}} \int_{\Omega_{t}^{\prime}} \rho_{\mathrm{f}} \phi d \Omega=\int_{\Omega_{t}^{\prime}} \theta d \Omega .
$$

Therefore, invoking Lemma 2 with $u=\rho_{\mathrm{f}} \phi$, i.e. $u_{m}=1$, we get

$$
\frac{\partial}{\partial t}\left(\rho_{\mathrm{f}} \phi\right)+\underline{\nabla}_{\underline{\mathrm{x}}} \cdot\left(\rho_{\mathrm{f}} \phi \underline{v}_{\mathrm{s}}+\underline{w}\right)=\theta .
$$

We point out that since $\rho_{\mathrm{f}} \phi$ is a fluid quantity we can also express the conservation law by the simple identity

$$
\frac{\mathrm{d}_{\mathrm{f}}}{\mathrm{dt}} \int_{\Omega_{t}^{\prime}}\left(\rho_{\mathrm{f}} \phi\right) d \Omega=\int_{\Omega_{t}^{\prime}} \theta d \Omega .
$$

Let us now consider the total mass conservation. Introducing the total density

$$
\rho=\rho_{\mathrm{s}}(1-\phi)+\rho_{\mathrm{f}} \phi,
$$

we have as in (8),

$$
\frac{\mathrm{D}}{\mathrm{Dt}} \int_{\Omega_{t}^{\prime}} \rho d \Omega=\int_{\Omega_{t}^{\prime}} \theta d \Omega .
$$

Then, taking $u=\rho$ in Lemma 2 - namely, still $u_{m}=1$ - we get

$$
\frac{\partial \rho}{\partial t}+\underline{\nabla}_{\underline{\mathrm{x}}} \cdot\left(\rho \underline{v}_{\mathrm{s}}+\underline{w}\right)=\theta .
$$

Hence, we obtain by subtraction the conservation law for the skeleton

$$
\frac{\partial}{\partial t}\left(\rho_{\mathrm{s}}(1-\phi)\right)+\underline{\nabla}_{\underline{\mathrm{x}}} \cdot\left(\rho_{\mathrm{S}}(1-\phi) \underline{v}_{\mathrm{s}}\right)=0 .
$$

Remark 2 - In the three relations (9), (13) and (14), the time derivative identity (1) allows to extract the density from the divergence term by simply substituting for $\frac{\partial}{\partial t}$ the time derivative with respect to the skeleton $\frac{\mathrm{d}}{\mathrm{dt}}$. For example, (9) gives

$$
\frac{\mathrm{d}}{\mathrm{dt}}\left(\rho_{\mathrm{f}} \phi\right)+\rho_{\mathrm{f}} \phi \underline{\nabla}_{\underline{\mathrm{x}}} \cdot \underline{v}_{\mathrm{s}}+\underline{\nabla}_{\underline{\mathrm{x}}} \cdot \underline{w}=\theta .
$$


We conclude this section by giving the corresponding mass conservation law in the Lagrangian framework attached to the skeleton. Let us denote by $\rho_{0}=\rho_{\mathrm{s} 0}\left(1-\phi_{0}\right)+\rho_{\mathrm{f} 0} \phi_{0}$ the density in the reference configuration $\Omega_{0}$. We define by $m$ the Lagrangian density of added mass that represents the local change of fluid mass at every point $\underline{\xi}$ in the domain

$$
\rho(\underline{\xi}, t) J(\underline{\xi}, t)=\rho_{0}(\underline{\xi})+m(\underline{\xi}, t) .
$$

Since the solid mass is conserved, we directly have

$$
m=\rho_{\mathrm{f}} J \phi-\rho_{\mathrm{f} 0} \phi_{0} .
$$

Using the differentiation formula in Lagrangian framework (7) with $U=\rho_{0}+m$ and $u_{m}=1$ we get

$$
\frac{\mathrm{d} m}{\mathrm{dt}}+\underline{\nabla_{\xi}} \cdot \underline{W}=\Theta,
$$

where $\Theta=J \theta$ is the Lagrangian source density associated with $\theta$.

\section{Principle of virtual work for the mixture}

In this section, we derive the principle of virtual work for the mixture, mainly based on the conservation of momentum.

\subsection{Expression of accelerations}

For the skeleton, the definition of the acceleration is straightforward and corresponds to

$$
\underline{\gamma}_{\mathrm{s}}=\frac{\mathrm{d} \underline{v}_{\mathrm{s}}}{\mathrm{dt}}=\frac{\partial \underline{v}_{\mathrm{s}}}{\partial t}+\underline{\nabla}_{\underline{\underline{v}}} \underline{v}_{\mathrm{s}} \cdot \underline{v}_{\mathrm{s}}
$$

but for the fluid we need to use the material derivative with respect to the fluid particles, hence we have

$$
\underline{\gamma}_{\mathrm{f}}=\frac{\mathrm{d}_{\mathrm{f}} \underline{\mathrm{f}}_{\mathrm{f}}}{\mathrm{dt}}=\frac{\partial \underline{v}_{\mathrm{f}}}{\partial t}+\underline{\nabla}_{\underline{\underline{ }}} \underline{v}_{\mathrm{f}} \cdot \underline{v}_{\mathrm{f}}=\underline{\gamma}_{\mathrm{s}}+\frac{\mathrm{d} \underline{v}_{r}}{\mathrm{dt}}+\underline{\underline{\nabla}}_{\underline{x}}\left(\underline{v}_{\mathrm{s}}+\underline{v}_{r}\right) \cdot \underline{v}_{r} .
$$

\subsection{Momentum conservation laws}

We now focus on the extension of the momentum conservation law to poromechanics systems. The system considered is open, hence we have for any sub-domain $\Omega_{t}^{\prime}$

$$
\frac{\mathrm{D}}{\mathrm{Dt}} \int_{\Omega_{t}^{\prime}}\left(\rho \underline{v}_{\mathrm{s}}+\rho_{\mathrm{f}} \phi \underline{v}_{r}\right) d \Omega=\int_{\Omega_{t}^{\prime}} \theta \underline{v}_{\mathrm{f}} d \Omega+\int_{\Omega_{t}^{\prime}} \rho \underline{f} d \Omega+\int_{\partial \Omega_{t}^{\prime}} \underline{t} d S,
$$

where $\theta \underline{v}_{\mathrm{f}}$ is the variation of momentum due to the fluid source term. Considering the left-hand side, we use Lemma 2 with $\underline{u}=\rho \underline{v}_{\mathrm{S}}+\rho_{\mathrm{f}} \phi \underline{v}_{r}$ and $\underline{u}_{m}=\underline{v}_{\mathrm{f}}=\underline{v}_{\mathrm{S}}+\underline{v}_{r}$ to obtain

$$
\begin{aligned}
\frac{\mathrm{D}}{\mathrm{Dt}} \int_{\Omega_{t}^{\prime}}\left(\rho \underline{v}_{\mathrm{s}}+\rho_{\mathrm{f}} \phi \underline{v}_{r}\right) d \Omega= & \int_{\Omega_{t}^{\prime}} \frac{\partial}{\partial t}\left(\rho \underline{v}_{\mathrm{S}}+\rho_{\mathrm{f}} \phi \underline{v}_{r}\right) d \Omega \\
& +\int_{\Omega_{t}^{\prime}}\left(\underline{\nabla}_{\underline{\mathrm{x}}} \cdot\left(\left(\rho \underline{v}_{\mathrm{s}}+\rho_{\mathrm{f}} \phi \underline{v}_{r}\right) \otimes \underline{v}_{\mathrm{s}}\right)+\underline{\nabla}_{\underline{\mathrm{x}}} \cdot\left(\left(\underline{v}_{\mathrm{s}}+\underline{v}_{r}\right) \otimes \underline{w}\right)\right) d \Omega .
\end{aligned}
$$


Developing the right hand side we get

$$
\begin{aligned}
& \frac{\partial}{\partial t}\left(\rho \underline{v}_{\mathrm{S}}+\rho_{\mathrm{f}} \phi \underline{v}_{r}\right)+\underline{\nabla}_{\underline{\mathrm{x}}} \cdot\left(\left(\rho \underline{v}_{\mathrm{S}}\right.\right.\left.\left.+\rho_{\mathrm{f}} \phi \underline{v}_{r}\right) \otimes \underline{v}_{\mathrm{S}}\right)+\underline{\nabla}_{\underline{\mathrm{x}}} \cdot\left(\left(\underline{v}_{\mathrm{S}}+\underline{v}_{r}\right) \otimes \underline{w}\right)= \\
&\left.\left.\rho\left(\frac{\partial \underline{v}_{\mathrm{S}}}{\partial t}+\underline{\nabla}_{\underline{\underline{v}}} \underline{v}_{\mathrm{S}} \cdot \underline{v}_{\mathrm{S}}\right)\right)+\rho_{\mathrm{f}} \phi\left(\frac{\partial \underline{v}_{r}}{\partial t}+\underline{\nabla}_{\underline{\mathrm{x}}} \underline{v}_{r} \cdot \underline{v}_{\mathrm{S}}+\underline{\nabla}_{\underline{\mathrm{x}}}\left(\underline{v}_{\mathrm{S}}+\underline{v}_{r}\right) \cdot \underline{v}_{r}\right)\right) \\
& \quad+\left(\frac{\partial \rho}{\partial t}+\underline{\nabla}_{\underline{\mathrm{x}}} \cdot\left(\rho \underline{v}_{\mathrm{S}}+\underline{w}\right)\right) \underline{v}_{\mathrm{S}}+\left(\frac{\partial}{\partial t}\left(\rho_{\mathrm{f}} \phi\right)+\underline{\nabla}_{\underline{\mathrm{x}}} \cdot\left(\rho_{\mathrm{f}} \phi \underline{v}_{\mathrm{S}}+\underline{w}_{)}\right) \underline{v}_{r} .\right.
\end{aligned}
$$

Therefore, using the mass conservation laws and the acceleration definitions, we obtain

$\frac{\partial}{\partial t}\left(\rho \underline{v}_{\mathrm{s}}+\rho_{\mathrm{f}} \phi \underline{v}_{r}\right)+\underline{\nabla}_{\underline{\mathrm{x}}} \cdot\left(\left(\rho \underline{v}_{\mathrm{s}}+\rho_{\mathrm{f}} \phi \underline{v}_{r}\right) \otimes \underline{v}_{\mathrm{s}}\right)+\underline{\nabla}_{\underline{\mathrm{x}}} \cdot\left(\left(\underline{v}_{\mathrm{s}}+\underline{v}_{r}\right) \otimes \underline{w}\right)=\rho_{\mathrm{s}}(1-\phi) \underline{\gamma}_{\mathrm{s}}+\rho_{\mathrm{f}} \phi \underline{\gamma}_{\mathrm{f}}+\theta\left(\underline{v}_{\mathrm{s}}+\underline{v}_{r}\right)$,

and the (first) momentum conservation law yields

$$
\int_{\Omega_{t}^{\prime}}\left(\rho_{\mathrm{s}}(1-\phi) \underline{\gamma}_{\mathrm{s}}+\rho_{\mathrm{f}} \phi \underline{\gamma}_{\mathrm{f}}\right) d \Omega=\int_{\Omega_{t}^{\prime}} \rho \underline{f} d \Omega+\int_{\partial \Omega_{t}^{\prime}} \underline{t} d S .
$$

\subsection{Principle of virtual work}

The fundamental law of dynamics is deduced from the momentum conservation law when classically introducing the Cauchy stress tensor $\underline{\underline{\sigma}}$, which gives

$$
\underline{\nabla}_{\underline{\mathrm{x}}} \cdot \underline{\underline{\sigma}}+\rho \underline{f}-\left(\rho_{\mathrm{s}}(1-\phi) \underline{\gamma}_{\mathrm{s}}+\rho_{\mathrm{f}} \phi \underline{\gamma}_{\mathrm{f}}\right)=0 \text {, in } \Omega_{t} .
$$

Remark 3 - We recall that $\underline{\underline{\sigma}}$ can be proven to be symmetric using the second momentum conservation law on the cross product between the position and the momentum (see e.g. [16]).

In order to simplify the writing of the principle of virtual work, let us define $\underline{\gamma}=\frac{1}{\rho}\left(\rho_{\mathrm{s}}(1-\right.$ $\left.\phi) \underline{\gamma}_{\mathrm{s}}+\rho_{\mathrm{f}} \phi \underline{\gamma}_{\mathrm{f}}\right)$. The weak formulation of the above fundamental law of dynamics is given on the space of admissible displacements $V\left(\Omega_{t}\right)$ by

$$
\forall \underline{v}^{*} \in V\left(\Omega_{t}\right), \quad \int_{\Omega_{t}} \underline{\underline{\sigma}}: \underline{\underline{\nabla}}_{\underline{\underline{v}}} \underline{v}^{*} d \Omega=\int_{\Omega_{t}} \rho(\underline{f}-\underline{\gamma}) \cdot \underline{v}^{*} d \Omega+\int_{\partial \Omega_{t}} \underline{t} \cdot \underline{v}^{*} d S .
$$

Considering the symmetry of the Cauchy stress tensor, we can symmetrize $\underline{\underline{\nabla}}_{\underline{x}}$ by using the linearized deformation tensor in the deformed configuration $\underline{\underline{\varepsilon}}\left(\underline{v}^{*}\right)$, so that

$$
\forall \underline{v}^{*} \in V\left(\Omega_{t}\right), \quad \int_{\Omega_{t}} \underline{\underline{\sigma}}: \underline{\underline{\varepsilon}}\left(\underline{v}^{*}\right) d \Omega=\int_{\Omega_{t}} \rho(\underline{f}-\underline{\gamma}) \cdot \underline{v}^{*} d \Omega+\int_{\partial \Omega_{t}} \underline{t} \cdot \underline{v}^{*} d S .
$$

The change of variable $\underline{x} \rightarrow \underline{\xi}$ allows to obtain an expression in the configuration attached to the skeleton. In fact,

$$
\underline{\underline{\nabla}}_{\underline{\underline{v}}} \underline{v}^{*}=\underline{\underline{\nabla}}_{\underline{\xi}} \underline{v}^{*} \cdot \underline{\underline{\nabla}} \underline{\underline{\xi}} \underline{\underline{\theta}}=\underline{\underline{\nabla}}_{\underline{\xi}} \underline{v}^{*} \cdot\left(\underline{\underline{\nabla}}_{\underline{\xi}}\right)^{-1}=\underline{\underline{\nabla}}_{\underline{\xi}} \underline{v}^{*} \cdot \underline{\underline{F}}^{-1}
$$

hence, we get from (22)

$\forall \underline{v}^{*} \in V\left(\Omega_{0}\right), \quad \int_{\Omega_{0}} \underline{\underline{\sigma}}: \underline{\underline{\nabla}}_{\underline{\underline{v}}}^{\underline{v}^{*}} \cdot \underline{\underline{F}}^{-1} J d \Omega=\int_{\Omega_{0}}\left(\rho_{0}+m\right)(\underline{f}-\underline{\gamma}) \cdot \underline{v}^{*} d \Omega+\int_{\partial \Omega_{0}} J\left\|\underline{\underline{F}}^{-T} \cdot \underline{n}_{0}\right\| \underline{t} \cdot \underline{v}^{*} d S$.

Considering the second Piola-Kirchhoff stress tensor in order to keep a symmetric tensor

$$
\underline{\underline{\underline{\Sigma}}}=J \underline{\underline{F}}^{-1} \cdot \underline{\underline{\sigma}} \cdot \underline{\underline{F}}^{-T},
$$


and introducing $\underline{t}_{0}=J\left\|\underline{\underline{F}}^{-T} \cdot \underline{n}_{0}\right\| \underline{t}$ the transported traction on the boundary, standard manipulations yield the corresponding Lagrangian form for the weak formulation of the equation of motion

$\forall \underline{v}^{*} \in V\left(\Omega_{0}\right), \quad \int_{\Omega_{0}}\left(\rho_{0}+m\right) \underline{\gamma} \cdot \underline{v}^{*} d \Omega+\int_{\Omega_{0}} \underline{\underline{\Sigma}}: d_{\underline{y}} \underline{\underline{e}} \cdot \underline{v}^{*} d \Omega=\int_{\Omega_{0}}\left(\rho_{0}+m\right) \underline{f} \cdot \underline{v}^{*} d \Omega+\int_{\partial \Omega_{0}} \underline{t}_{0} \cdot \underline{v}^{*} d S$,

with $d_{y} \underline{\underline{e}}$ the differential of $\underline{\underline{e}}$ given by

$$
d_{\underline{y}} \underline{\underline{e}} \cdot \underline{v}^{*}=\frac{1}{2}\left(\underline{\underline{F}}^{T} \cdot \underline{\nabla}_{\underline{\xi}} \underline{v}^{*}+\left(\underline{\nabla}_{\underline{\xi}} \underline{v}^{*}\right)^{T} \cdot \underline{\underline{F}}\right),
$$

and the associated strong formulation is

$$
\underline{\nabla_{\underline{\xi}}} \cdot(\underline{\underline{F}} \cdot \underline{\underline{\Sigma}})+\left(\rho_{0}+m\right)(\underline{f}-\underline{\gamma})=0, \text { in } \Omega_{0}
$$

The weak formulation, also called principle of virtual work, can be finally summarized as

$$
\forall \underline{v}^{*} \in V(\Omega), \quad \mathcal{P}_{i}\left(\underline{v}^{*}\right)+\mathcal{P}_{\text {int }}\left(\underline{v}^{*}\right)=\mathcal{P}_{\text {ext }}\left(\underline{v}^{*}\right),
$$

with

$$
\left\{\begin{array}{l}
\mathcal{P}_{i}\left(\underline{v}^{*}\right)=\int_{\Omega_{t}}\left(\rho_{\mathrm{s}}(1-\phi) \underline{\gamma}_{\mathrm{s}}+\rho_{\mathrm{f}} \phi \underline{\gamma}_{\mathrm{f}}\right) \cdot \underline{v}^{*} d \Omega=\int_{\Omega_{0}}\left(\rho_{\mathrm{s} 0}\left(1-\phi_{0}\right) \underline{\gamma}_{\mathrm{s}}+\left(\rho_{\mathrm{f} 0} \phi_{0}+m\right) \underline{\gamma}_{\mathrm{f}}\right) \cdot \underline{v}^{*} d \Omega \\
\mathcal{P}_{\mathrm{int}}\left(\underline{v}^{*}\right)=\int_{\Omega_{t}} \underline{\underline{\sigma}}: \underline{\underline{\varepsilon}}\left(\underline{v}^{*}\right) d \Omega=\int_{\Omega_{0}} \underline{\underline{\Sigma}}: d_{\underline{y}} \underline{\underline{e}} \cdot \underline{v}^{*} d \Omega \\
\mathcal{P}_{\text {ext }}\left(\underline{v}^{*}\right)=\int_{\Omega_{t}} \rho \underline{f} \cdot \underline{v}^{*} d \Omega+\int_{\partial \Omega_{t}} \underline{t} \cdot \underline{v}^{*} d S=\int_{\Omega_{0}}\left(\rho_{0}+m\right) \underline{f} \cdot \underline{v}^{*} d \Omega+\int_{\partial \Omega_{0}} \underline{t}_{0} \cdot \underline{v}^{*} d S
\end{array}\right.
$$

\section{Kinetic energy and first law of thermodynamics}

The objective of this section is to express the first law of thermodynamics applied to the poromechanics system considered.

\subsection{Kinetic energy theorem}

By definition, the kinetic energy of the mixture is

$$
\begin{aligned}
\mathcal{K}=\mathcal{K}_{s}+\mathcal{K}_{\mathrm{f}} & =\frac{1}{2} \int_{\Omega_{t}}\left(\rho_{\mathrm{s}}(1-\phi) \underline{v}_{\mathrm{s}}^{2}+\rho_{\mathrm{f}} \phi \underline{v}_{\mathrm{f}}^{2}\right) d \Omega \\
& =\frac{1}{2} \int_{\Omega_{0}}\left(\rho_{0}\left(1-\phi_{0}\right) \underline{v}_{\mathrm{s}}^{2}+\left(\rho_{\mathrm{f} 0} \phi_{0}+m\right) \underline{v}_{\mathrm{f}}^{2}\right) d \Omega .
\end{aligned}
$$

We then have the following identity.

\section{Lemma 3}

When considering the total derivative of the mixture kinetic energy, we have

$$
\begin{aligned}
\frac{\mathrm{DK}}{\mathrm{Dt}} & =\int_{\Omega_{t}}\left(\left(\rho_{\mathrm{s}}(1-\phi) \underline{\gamma}_{\mathrm{s}}+\rho_{\mathrm{f}} \phi \underline{\gamma}_{\mathrm{f}}\right) \cdot \underline{v}_{\mathrm{s}}+\rho_{\mathrm{f}} \phi \underline{\gamma}_{\mathrm{f}} \cdot \underline{v}_{r}+\frac{1}{2} \theta \underline{v}_{\mathrm{f}}^{2}\right) d \Omega \\
& =\int_{\Omega_{0}}\left(\left(\rho_{\mathrm{s} 0}\left(1-\phi_{0}\right) \underline{\gamma}_{\mathrm{s}}+\rho_{\mathrm{f} 0} \phi_{0} \underline{\gamma}_{\mathrm{f}}\right) \cdot \underline{v}_{\mathrm{s}}+\left(\rho_{\mathrm{f} 0} \phi_{0}+m\right) \underline{\gamma}_{\mathrm{f}} \cdot \underline{v}_{r}+\frac{1}{2} \Theta \underline{v}_{\mathrm{f}}^{2}\right) d \Omega_{0}
\end{aligned}
$$


Proof. Straightforward by noting that

$$
\frac{\mathrm{d}_{\mathrm{f}}}{\mathrm{dt}} \int_{\Omega_{t}} \frac{1}{2} \rho_{\mathrm{f}} \phi \underline{v}_{\mathrm{f}}^{2} d \Omega=\int_{\Omega_{t}} \frac{1}{2}\left(\underline{v}_{\mathrm{f}}^{2} \frac{\mathrm{d}_{\mathrm{f}}}{\mathrm{dt}}\left(\rho_{\mathrm{f}} \phi d \Omega\right)+\rho_{\mathrm{f}} \phi \frac{\mathrm{d}_{\mathrm{f}} \underline{v}_{\mathrm{f}}^{2}}{\mathrm{dt}} d \Omega\right)=\int_{\Omega_{t}}\left(\frac{1}{2} \theta \underline{v}_{\mathrm{f}}^{2}+\rho_{\mathrm{f}} \phi \underline{\gamma}_{\mathrm{f}} \cdot \underline{v}_{\mathrm{f}}\right) d \Omega,
$$

using the fluid mass conservation (10).

Therefore, introducing the contribution of the fluid in the inertial virtual work

$$
\mathcal{P}_{i}^{\mathrm{f}}\left(\underline{v}^{*}\right)=\int_{\Omega_{t}} \rho_{\mathrm{f}} \phi \underline{\gamma}_{\mathrm{f}} \cdot \underline{v}^{*} d \Omega,
$$

and the kinetic energy variation due to the source term

$$
\mathcal{J}_{\mathcal{K} \theta}=\frac{1}{2} \int_{\Omega_{t}} \theta \underline{v}_{\mathrm{f}}^{2} d \Omega
$$

we have the following result directly obtained by combining (25) applied with $\underline{v}^{*}=\underline{v}_{\mathrm{S}}$ and (28).

\section{Theorem 4 (Kinetic Energy Theorem)}

$$
\frac{\mathrm{D} \mathcal{K}}{\mathrm{Dt}}-\mathcal{P}_{i}^{\mathrm{f}}\left(\underline{v}_{r}\right)+\mathcal{P}_{\text {int }}\left(\underline{v}_{\mathrm{s}}\right)=\mathcal{J}_{\mathcal{K} \theta}+\mathcal{P}_{\text {ext }}\left(\underline{v}_{\mathrm{s}}\right)
$$

\subsection{First law of thermodynamics}

Let us introduce the internal energy and its corresponding volume-distributed form

$$
\mathcal{E}=\int_{\Omega_{t}} e d \Omega
$$

Part of the internal energy is associated with the fluid phase, which is represented by a fluid energy per unit mass $e_{m}$. As is standard in fluid thermodynamics, we also introduce the fluid enthalpy per unit mass, defined by

$$
h_{m}=e_{m}+\frac{p}{\rho_{\mathrm{f}}}
$$

and

$$
\mathcal{J}_{\mathcal{H}_{\theta}}=\int_{\Omega_{t}} \theta h_{m} d \Omega
$$

corresponds to the variation of enthalpy due to the source term [38]. Finally, we denote the external work specifically applied on the fluid by

$$
\mathcal{P}_{\text {ext }}^{\mathrm{f}}\left(\underline{v}^{*}\right)=\int_{\Omega_{t}} \rho_{\mathrm{f}} \phi \underline{f} \cdot \underline{v}^{*} d \Omega+\int_{\partial \Omega_{t}} \phi\left(\underline{\underline{\sigma}}_{\mathrm{f}} \cdot \underline{n}\right) \cdot \underline{v}^{*} d S
$$

where $\underline{\sigma}_{\mathrm{f}}$ is the fluid Cauchy stress tensor, with the usual decomposition into pressure and viscous parts, namely,

$$
\underline{\underline{\sigma}}_{\mathrm{f}}=\underline{\underline{\sigma}}_{\mathrm{vis}}\left(\underline{v}_{\mathrm{f}}\right)-p \underline{\underline{\mathbb{1}}}
$$

such that when applied to the relative velocity, we obtain the following power

$$
\begin{aligned}
\mathcal{P}_{\mathrm{ext}}^{\mathrm{f}}\left(\underline{v}_{r}\right) & =\int_{\Omega_{t}} \underline{f} \cdot \underline{w} d \Omega-\int_{\partial \Omega_{t}} \frac{\underline{p}}{\rho_{\mathrm{f}}} \underline{n} \cdot \underline{w} d S+\int_{\partial \Omega_{t}} \frac{1}{\rho_{\mathrm{f}}}\left(\underline{\underline{\sigma}}_{\mathrm{vis}} \cdot \underline{n}\right) \cdot \underline{w} d S \\
& =\int_{\Omega_{t}} \underline{f} \cdot \underline{w} d \Omega+\int_{\Omega_{t}} \underline{\nabla}_{\underline{\mathrm{x}}} \cdot\left(\frac{1}{\rho_{\mathrm{f}}} \underline{\sigma}_{\mathrm{f}} \cdot \underline{w}\right) d \Omega .
\end{aligned}
$$




\section{Principle 1 (First law of thermodynamics)}

For an open system, the variation of the total energy of the system - which is the sum of the internal energy and the kinetic energy - equals the power of the external loads added to the heat rate $\mathcal{Q}$, and to the rates of kinetic energy and enthalpy due to the source terms, namely,

$$
\frac{\mathrm{D} \mathcal{E}_{T}}{\mathrm{Dt}}=\frac{\mathrm{D} \mathcal{K}}{\mathrm{Dt}}+\frac{\mathrm{D} \mathcal{E}}{\mathrm{Dt}}=\mathcal{J}_{\mathcal{K}_{\theta}}+\mathcal{J}_{\mathcal{H}_{\theta}}+\mathcal{P}_{\text {ext }}\left(\underline{v}_{\mathrm{s}}\right)+\mathcal{P}_{\text {ext }}^{\mathrm{f}}\left(\underline{v}_{r}\right)+\mathcal{Q} .
$$

Remark 4 - As already seen for several integrated quantities, we can decompose the power of external forces into two contributions, the first one given here by the power of forces working on a global velocity field $\underline{v}_{\mathrm{S}}$ considered on the whole mixture, and the second one for forces acting only on the fluid phase and working on the relative velocity $\underline{v}_{r}$, namely,

$$
\mathcal{P}_{\text {ext }}^{\text {total }}=\mathcal{P}_{\text {ext }}\left(\underline{v}_{\mathrm{S}}\right)+\mathcal{P}_{\text {ext }}^{\mathrm{f}}\left(\underline{v}_{r}\right) .
$$

The heat rate can be decomposed into the volume-distributed heat source term and the heat flux at the boundaries as follows

$$
\begin{aligned}
\mathcal{Q} & =\int_{\Omega_{t}} q d \Omega-\int_{\partial \Omega_{t}} \underline{j}_{q} \cdot \underline{n} d S \\
& =\int_{\Omega_{0}} Q d \Omega-\int_{\partial \Omega_{0}} \underline{J}_{Q} \cdot \underline{n}_{0} d S,
\end{aligned}
$$

with $\underline{J}_{Q}=J \underline{\underline{F}}^{-1} \underline{j}_{q}$. We can then apply the differentiation lemma to the volume-distributed internal energy $e$, and define $e_{m}$ such that

$$
\frac{\mathrm{D} \mathcal{E}}{\mathrm{Dt}}=\int_{\Omega_{t}}\left(\frac{\partial e}{\partial t}+\underline{\nabla}_{\underline{\mathbf{x}}} \cdot\left(e \underline{v}_{\mathrm{s}}\right)+\underline{\nabla}_{\underline{\mathbf{x}}} \cdot\left(e_{m} \underline{w}\right)\right) d \Omega
$$

and - as in fact the first principle can be applied to any subsystem associated with a subdomain $\Omega_{t}^{\prime} \subset \Omega_{t}-$ we infer the corresponding local formulation using (31)

$$
\frac{\partial e}{\partial t}+\underline{\nabla}_{\underline{\mathrm{X}}} \cdot\left(e \underline{v}_{\mathrm{s}}\right)=\theta h_{m}-\underline{\nabla}_{\underline{\mathrm{X}}} \cdot\left(h_{m} \underline{w}\right)+\underline{\underline{\sigma}}: \underline{\underline{\varepsilon}}\left(\underline{v}_{\mathrm{s}}\right)+\underline{\nabla}_{\underline{\mathrm{X}}} \cdot\left(\frac{1}{\rho_{\mathrm{f}}} \underline{\sigma}_{\mathrm{vis}} \cdot \underline{w}\right)+\underline{w} \cdot\left(\underline{f}-\underline{\gamma}_{\mathrm{f}}\right)+q-\underline{\nabla}_{\underline{\mathrm{x}}} \cdot \underline{j}_{q},
$$

or equivalently

$$
\frac{\mathrm{d} e}{\mathrm{dt}}+e \underline{\nabla}_{\underline{\mathrm{x}}} \cdot \underline{v}_{\mathrm{s}}=\theta h_{m}-\underline{\nabla}_{\underline{\mathrm{x}}} \cdot\left(h_{m} \underline{w}\right)+\underline{\underline{\sigma}}: \underline{\underline{\varepsilon}}\left(\underline{v}_{\mathrm{s}}\right)+\underline{\nabla}_{\underline{\mathrm{x}}} \cdot\left(\frac{1}{\rho_{\mathrm{f}}} \underline{\underline{\sigma}} \cdot \underline{\mathrm{vis}} \cdot \underline{w}\right)+\underline{w} \cdot\left(\underline{f}-\underline{\gamma}_{\mathrm{f}}\right)+q-\underline{\nabla}_{\underline{\mathrm{x}}} \cdot \underline{j}_{q},
$$

which also gives in the total Lagrangian framework

$$
\frac{\mathrm{d} E}{\mathrm{dt}}=\Theta h_{m}-\underline{\nabla}_{\underline{\xi}} \cdot\left(h_{m} \underline{W}\right)+\underline{\underline{\Sigma}}:\left(d_{\underline{y}} \underline{\underline{e}} \cdot \underline{v}_{\mathrm{s}}\right)+\underline{\nabla}_{\underline{\xi}} \cdot\left(\frac{1}{\rho_{\mathrm{f}} J} \underline{\underline{\Pi}}_{\mathrm{vis}}^{T} \cdot \underline{\underline{F}} \cdot \underline{W}\right)+(\underline{\underline{F}} \cdot \underline{W}) \cdot\left(\underline{f}-\underline{\gamma}_{\mathrm{f}}\right)+Q-\underline{\nabla}_{\underline{\xi}} \cdot \underline{J}_{Q},
$$

with $\underline{\underline{M}}_{\mathrm{vis}}$ the first Piola-Kirchhoff stress tensor with respect to the skeleton configuration for the fluid viscous stresses, namely,

$$
\underline{\underline{\Pi}}_{\mathrm{vis}}=J \underline{\underline{\sigma}}_{\mathrm{vis}} \cdot \underline{\underline{F}}^{-T} \text {. }
$$

\section{Constitutive laws derived from the second principle}

In this section, we proceed to apply the second law of thermodynamics, which will provide Clausius-Duhem inequalities allowing to infer adequate constitutive laws. 


\subsection{Second law of thermodynamics}

As we did for the internal energy, we introduce the entropy and its corresponding volumedistributed form

$$
\mathcal{S}=\int_{\Omega_{t}} s d \Omega
$$

and the entropy rate due to the source term

$$
\mathcal{J}_{\mathcal{S}_{\theta}}=\int_{\Omega_{t}} \theta s_{m} d \Omega
$$

\section{Principle 2 (Second law of thermodynamics)}

Let us consider an open system within a temperature field $T$, and submitted to a heat source $q$ and a heat flux $\underline{j}_{q}$, then we have

$$
\frac{\mathrm{DS}}{\mathrm{Dt}} \geq \mathcal{J}_{\mathcal{S}_{\theta}}+\int_{\Omega_{t}} \frac{q}{T} d \Omega-\int_{\partial \Omega_{t}} \frac{\underline{j} \cdot \underline{n}}{T} d S=\int_{\Omega_{t}}\left(\theta s_{m}+\frac{q}{T}-\underline{\nabla}_{\underline{\mathbf{x}}} \cdot\left(\frac{\underline{j}}{T}\right)\right) d \Omega .
$$

As we have done in the first principle, we can convert this into a space-distributed inequality, namely,

$$
\frac{\mathrm{d} s}{\mathrm{dt}}+s \underline{\nabla}_{\underline{\mathrm{x}}} \cdot \underline{v}_{\mathrm{s}} \geq \theta s_{m}-\underline{\nabla}_{\underline{\mathrm{x}}} \cdot\left(s_{m} \underline{w}\right)+\frac{q}{T}-\frac{1}{T} \underline{\nabla}_{\underline{\mathrm{x}}} \cdot \underline{j}_{q}+\frac{1}{T^{2}} \underline{j}_{q} \cdot \underline{\nabla}_{\underline{\mathrm{x}}} T .
$$

In order to deal with thermodynamics functions of $T$ and other variables - $\underline{\underline{e}}$ and $m$ for the fluid-solid mixture and $p$ for the fluid - we classically introduce

- for the fluid-solid mixture, the Helmholtz free energy

$$
\psi(\underline{\underline{e}}, m, T)=e(\underline{\underline{e}}, m, s)-T s,
$$

- for the fluid, the Gibbs free energy (sometimes also called free enthalpy)

$$
g_{m}(p, T)=h_{m}\left(p, s_{m}\right)-T s_{m}
$$

As usual, we will also consider the Lagrangian counterparts of such quantities, namely densities per unit volume in the reference configuration of the skeleton, denoted by upper case letters such as $S$ or $\Psi$. We also recall the fundamental identities (see e.g. [16])

$$
\frac{\partial g_{m}}{\partial p}=\frac{1}{\rho_{\mathrm{f}}}, \quad \frac{\partial g_{m}}{\partial T}=-s_{m}
$$

the first of which being the state equation of the fluid.

Remark 5 - It is very interesting - and by no means straightforward - that the internal energy and the Helmholtz free energy of the mixture only depend on the above compact sets of variables. Indeed, for example we have for the Helmholtz free energy in Lagrangian form

$$
\Psi=\Psi_{\mathrm{s}}(\underline{\underline{e}}, J(1-\phi), T)+m_{\mathrm{f}} \psi_{m}\left(\frac{1}{\rho_{\mathrm{f}}}, T\right),
$$

where $\Psi_{\mathrm{s}}$ is the free energy of the skeleton (per unit volume of the reference configuration) which satisfies

$$
d \Psi_{\mathrm{s}}=\underline{\underline{\bar{\Sigma}}}: d \underline{\underline{e}}-p d(J(1-\phi))-S_{\mathrm{s}} d T
$$


with $\underline{\underline{\underline{\Sigma}}}=\frac{\partial \Psi_{\mathrm{s}}}{\partial \underline{\underline{e}}}$, while $m_{\mathrm{f}}=m+\rho_{\mathrm{f} 0} \phi_{0}$ and $\psi_{m}$ is the Helmholtz free energy of the fluid per unit mass with

$$
d \psi_{m}=-p d\left(\frac{1}{\rho_{\mathrm{f}}}\right)-s_{\mathrm{f}} d T .
$$

We infer, recalling the identity $\psi_{m}=g_{m}-\frac{p}{\rho_{\mathrm{f}}}$,

$$
\begin{aligned}
d \Psi & =d \Psi_{\mathrm{s}}+m_{\mathrm{f}} d \psi_{m}+\psi_{m} d m \\
& =\underline{\underline{\Sigma}}: d \underline{\underline{e}}-p d J+p d(J \phi)-m_{\mathrm{f}} p d\left(\frac{1}{\rho_{\mathrm{f}}}\right)+\left(g_{m}-\frac{p}{\rho_{\mathrm{f}}}\right) d m-S d T \\
& =\underline{\underline{\Sigma}}^{t o t}: d \underline{\underline{e}}+g_{m} d m-S d T,
\end{aligned}
$$

with $\underline{\underline{\Sigma}}^{t o t}=\underline{\underline{\Sigma}}-p J \underline{\underline{C}}^{-1}$, and where the last simplification directly follows from the identity $m_{\mathrm{f}} \frac{1}{\rho_{\mathrm{f}}}=J \phi$, which shows the expected result, and further establishes the fundamental identities

$$
\frac{\partial \Psi}{\partial T}=-S, \quad \frac{\partial \Psi}{\partial m}=g_{m} .
$$

We can now combine (36) and (38) to eliminate $\left(q-\underline{\nabla}_{\underline{\mathrm{x}}} \cdot \underline{j}_{q}\right)$, which yields

$$
\begin{aligned}
\underline{\underline{\sigma}}: \underline{\underline{\varepsilon}}\left(\underline{v}_{\mathrm{s}}\right)+\underline{w}\left(\underline{f}-\underline{\gamma}_{\mathrm{f}}\right)+\underline{\nabla}_{\underline{\mathrm{x}}} \cdot\left(\frac{1}{\rho_{\mathrm{f}}} \underline{\underline{\sigma}} \cdot \underline{\mathrm{vis}} \cdot \underline{w}\right) \\
\quad+\theta g_{m}-\frac{\mathrm{d} \psi}{\mathrm{dt}}-\psi \underline{\nabla}_{\underline{\mathrm{x}}} \cdot \underline{v}_{\mathrm{s}}-\underline{\nabla}_{\underline{\mathrm{x}}} \cdot\left(h_{m} \underline{w}\right)+T \underline{\nabla}_{\underline{\mathrm{x}}} \cdot\left(s_{m} \underline{w}\right)-s \frac{\mathrm{d} T}{\mathrm{dt}}-\frac{\underline{j}_{q}}{T} \cdot \underline{\nabla}_{\underline{\mathrm{x}}} T \geq 0 .
\end{aligned}
$$

Then, using the Gibbs free energy $g_{m}$ that satisfies from (39)

$$
\underline{\nabla}_{\underline{\mathrm{x}}} g_{m}=\frac{\partial g_{m}}{\partial p} \underline{\nabla}_{\underline{\mathrm{x}}} p+\frac{\partial g_{m}}{\partial T} \underline{\nabla}_{\underline{\mathrm{x}}} T=\frac{1}{\rho_{\mathrm{f}}} \underline{\nabla}_{\underline{\mathrm{x}}} p-s_{m} \underline{\nabla}_{\underline{\mathrm{x}}} T
$$

we get, also substituting $\theta$ using the mass conservation (9),

$$
\begin{aligned}
\underline{\underline{\sigma}}: \underline{\underline{\varepsilon}}\left(\underline{v}_{\mathrm{s}}\right)+\underline{\nabla}_{\underline{\mathrm{x}}} \cdot\left(\frac{1}{\rho_{\mathrm{f}}} \underline{\underline{\sigma}}=\underline{\mathrm{vis}} \cdot \underline{w}\right)+g_{m}\left(\frac{\partial \rho_{\mathrm{f}} \phi}{\partial t}+\underline{\nabla}_{\underline{\mathrm{x}}} \cdot\left(\rho_{\mathrm{f}} \phi \underline{v}_{\mathrm{s}}\right)\right) \\
\quad-\frac{\mathrm{d} \psi}{\mathrm{dt}}-\psi \underline{\nabla}_{\underline{\mathrm{x}}} \cdot \underline{v}_{\mathrm{s}}-s \frac{\mathrm{d} T}{\mathrm{dt}}+\frac{\underline{w}}{\rho_{\mathrm{f}}}\left(-\underline{\nabla}_{\underline{\mathrm{x}}} p+\rho_{\mathrm{f}}\left(\underline{f}-\underline{\gamma}_{\mathrm{f}}\right)\right)-\frac{\underline{j}_{q}}{T} \cdot \underline{\nabla}_{\underline{\mathrm{x}}} T \geq 0,
\end{aligned}
$$

which can be rearranged into

$$
\begin{aligned}
\underline{\underline{\sigma}} & : \underline{\underline{\varepsilon}}\left(\underline{v}_{\mathrm{s}}\right)+\phi \underline{\underline{\sigma}}_{\mathrm{vis}}: \underline{\underline{\varepsilon}}\left(\underline{v}_{r}\right)+g_{m}\left(\frac{\partial \rho_{\mathrm{f}} \phi}{\partial t}+\underline{\nabla}_{\underline{\mathrm{x}}} \cdot\left(\rho_{\mathrm{f}} \phi \underline{v}_{\mathrm{s}}\right)\right) \\
& -\frac{\mathrm{d} \psi}{\mathrm{dt}}-\psi \underline{\nabla}_{\underline{\mathrm{x}}} \cdot \underline{v}_{\mathrm{s}}-s \frac{\mathrm{d} T}{\mathrm{dt}}+\frac{\underline{w}}{\rho_{\mathrm{f}}}\left(-\underline{\nabla}_{\underline{\mathrm{x}}} p+\rho_{\mathrm{f}}\left(\underline{f}-\underline{\gamma}_{\mathrm{f}}\right)+\frac{1}{\phi} \underline{\nabla}_{\underline{\mathrm{x}}} \cdot\left(\phi \underline{\sigma}_{\mathrm{vis}}\right)\right)-\frac{\underline{j}_{q}}{T} \cdot \underline{\nabla}_{\underline{\mathrm{x}}} T \geq 0,
\end{aligned}
$$

or in a Lagrangian framework using the Lagrangian counterpart of each quantity

$$
\begin{aligned}
& \underline{\underline{\Sigma}}:\left(\mathrm{d}_{\underline{y}} \underline{\underline{e}} \cdot \underline{v}_{\mathrm{s}}\right)+\phi \underline{\underline{\Sigma}}_{\mathrm{vis}}:\left(\mathrm{d}_{\underline{y}} \underline{\underline{e}} \cdot \underline{v}_{r}\right)+g_{m} \frac{\mathrm{d} m}{\mathrm{dt}} \\
& -\frac{\mathrm{d} \Psi}{\mathrm{dt}}-S \frac{\mathrm{d} T}{\mathrm{dt}}+\frac{\underline{W}}{\rho_{\mathrm{f}}} \cdot\left(-\underline{\nabla}_{\underline{\xi}} p+\rho_{\mathrm{f}} \underline{\underline{F}}^{T} \cdot\left(\underline{f}-\underline{\gamma}_{\mathrm{f}}\right)+\frac{1}{\phi} \underline{F}^{T} \cdot \underline{\nabla}_{\underline{\xi}} \cdot\left(\phi \underline{\underline{\Pi}}_{\mathrm{vis}}\right)\right)-\frac{\underline{J}_{Q}}{T} \cdot \underline{\nabla}_{\underline{\xi}} T \geq 0 .
\end{aligned}
$$

These inequalities are known as "Clausius-Duhem inequalities", another version of which can be obtained by noting that, here for the Lagrangian form,

$$
\frac{\mathrm{d} \Psi}{\mathrm{dt}}=\frac{\partial \Psi}{\partial \underline{\underline{e}}}: \mathrm{d}_{\underline{y}} \underline{\underline{e}} \cdot \underline{v}_{\mathrm{s}}+\frac{\partial \Psi}{\partial m} \frac{\mathrm{d} m}{\mathrm{dt}}+\frac{\partial \Psi}{\partial T} \frac{\mathrm{d} T}{\mathrm{dt}}=\frac{\partial \Psi}{\partial \underline{\underline{e}}}: \mathrm{d}_{\underline{y}} \underline{\underline{e}} \cdot \underline{v}_{\mathrm{s}}+g_{m} \frac{\mathrm{d} m}{\mathrm{dt}}-S \frac{\mathrm{d} T}{\mathrm{dt}},
$$

recalling (41). Hence, we can rewrite the inequality (44) in the following manner. 


\section{Theorem 5 (Clausius-Duhem inequality)}

$$
\begin{aligned}
\left(\underline{\underline{\Sigma}}-\frac{\partial \Psi}{\partial \underline{\underline{e}}}\right):\left(\mathrm{d}_{\underline{y}} \underline{\underline{e}} \cdot \underline{v}_{\mathrm{s}}\right)+\phi \underline{\underline{\Sigma}}_{\mathrm{vis}}:\left(\mathrm{d}_{\underline{y}} \underline{\underline{e}} \cdot \underline{v}_{r}\right) \\
\quad+\frac{\underline{\underline{W}}}{\rho_{\mathrm{f}}} \cdot\left(-\underline{\nabla}_{\underline{\xi}} p+\rho_{\mathrm{f}} \underline{\underline{F}}^{T} \cdot\left(\underline{f}-\underline{\gamma}_{\mathrm{f}}\right)+\frac{1}{\phi} \underline{F}^{T} \cdot \underline{\nabla}_{\underline{\xi}} \cdot\left(\phi \underline{\underline{\Pi}}_{\mathrm{vis}}\right)\right)-\frac{\underline{J}_{Q}}{T} \cdot \underline{\nabla}_{\underline{\xi}} T \geq 0 .
\end{aligned}
$$

\subsection{Constitutive laws}

Clausius-Duhem inequalities can be seen as providing the crucial conditions for devising constitutive laws, for which the second principle of thermodynamics should be satisfied. We therefore use (45) to infer the following result.

\section{Theorem 6}

The second principle is verified with the following conditions:

- For the heat rate, we suppose that Fourier's law is satisfied, namely,

$$
\underline{J}_{Q}=-\lambda_{Q} \underline{\nabla}_{\underline{\xi}} T \text {. }
$$

- For the stress tensor, we assume it is the sum of the three contributions

$$
\underline{\underline{\Sigma}}=\phi \underline{\underline{\Sigma}}_{\mathrm{vis}}+\frac{\partial \Psi}{\partial \underline{\underline{e}}}+\frac{\partial \Psi_{\text {damp }}}{\partial \underline{\underline{\dot{e}}}}
$$

with $\Psi^{\text {damp }}(\underline{\underline{e}}, \underline{\underline{e}})$ a viscous pseudo-potential convex in the variable $\underline{\underline{e}}=\frac{\mathrm{d}}{\mathrm{d}} \overline{\mathrm{e}}=\mathrm{d}_{\underline{y}} \underline{\underline{e}} \cdot \underline{v}_{\mathrm{s}}$ and satisfying $\frac{\partial \Psi_{\text {damp }}}{\partial \underline{\underline{e}}}(\underline{\underline{e}}, \underline{\underline{0}})=\underline{\underline{0}}$, which entails

$$
\frac{\partial \Psi_{\text {damp }}}{\partial \underline{\underline{\dot{e}}}}: \underline{\underline{\dot{e}}} \geq 0
$$

- For the fluid viscous term, we assume we always have the dissipation inequality

$$
\underline{\underline{\sigma}}_{\text {vis }}: \underline{\underline{\varepsilon}}\left(\underline{v}_{\mathrm{f}}\right) \geq 0 \text {. }
$$

- For the relative velocity of the fluid, there exists a positive definite second order tensor $\underline{\underline{K}}_{\mathrm{f}}$ such that

$$
\frac{1}{\rho_{\mathrm{f}}} \underline{W}=\underline{\underline{K}}_{\mathrm{f}} \cdot\left(-\underline{\nabla}_{\underline{\xi}} p+\rho_{\mathrm{f}} \underline{\underline{F}}^{T} \cdot\left(\underline{f}-\underline{\gamma}_{\mathrm{f}}\right)+\frac{1}{\phi} \underline{F}^{T} \cdot \underline{\nabla}_{\underline{\xi}} \cdot\left(\phi \underline{\underline{\Pi}}_{\mathrm{vis}}\right)\right),
$$

meaning in the Eulerian framework that

$$
\frac{1}{\rho_{\mathrm{f}}} \underline{w}=\underline{k}_{\mathrm{f}} \cdot\left(-\underline{\nabla}_{\underline{\mathrm{x}}} p+\rho_{\mathrm{f}}\left(\underline{f}-\underline{\gamma}_{\mathrm{f}}\right)+\frac{1}{\phi} \underline{\nabla}_{\underline{\mathrm{x}}} \cdot\left(\phi \underline{\underline{\sigma}}_{\text {vis }}\right)\right),
$$

with $\underline{\underline{k}}_{\mathrm{f}}=\frac{1}{J} \underline{\underline{F}} \cdot \underline{\underline{K}}_{\mathrm{f}} \cdot \underline{\underline{F}}^{T}$.

Proof. We easily verify that, under the assumptions made,

$$
\begin{gathered}
\left(\underline{\underline{\Sigma}}-\frac{\partial \Psi}{\partial \underline{\underline{e}}}\right): \mathrm{d}_{\underline{y}} \underline{\underline{e}} \cdot \underline{v}_{\mathrm{s}}+\phi \underline{\underline{\Sigma}}_{\mathrm{vis}}: \mathrm{d}_{\underline{y}} \underline{\underline{e}} \cdot \underline{v}_{r}+\frac{\underline{W}}{\rho_{\mathrm{f}}} \cdot\left(-\underline{\nabla}_{\underline{\xi}} p+\rho_{\mathrm{f}} \underline{\underline{F}}^{T} \cdot\left(\underline{f}-\underline{\gamma}_{\mathrm{f}}\right)+\frac{1}{\phi} \underline{F}^{T} \cdot \underline{\nabla}_{\underline{\xi}} \cdot\left(\phi \underline{\underline{\Pi}}_{\mathrm{vis}}\right)\right) \\
-\frac{\underline{J}_{Q}}{T} \cdot \underline{\nabla}_{\underline{\xi}} T=\frac{1}{J} \underline{\sigma}_{\mathrm{vis}}: \underline{\underline{\varepsilon}}\left(\underline{v}_{\mathrm{f}}\right)+\frac{\partial \Psi_{\mathrm{damp}}}{\partial \underline{\underline{\dot{e}}}}: \underline{\underline{e}}+\frac{\underline{W}}{\rho_{\mathrm{f}}} \cdot \underline{\underline{K}}_{\mathrm{f}}^{-1} \cdot \frac{\underline{W}}{\rho_{\mathrm{f}}}+\lambda_{Q} \underline{\nabla}_{\underline{\xi}} T \cdot \underline{\nabla}_{\underline{\xi}} T \geq 0 .
\end{gathered}
$$


Note that (46) can be seen as a generalized form of Darcy's law, which would correspond to the pressure gradient term alone. The contribution of the viscous stresses is often referred to as the Brinkman term, but the second principle leads to this most general form including also inertia and external force effects, see also [48] for a survey of such laws. The tensors $\underline{\underline{k}}_{\mathrm{f}}$ and $\underline{\underline{K}}_{\mathrm{f}}$ are called permeability tensors, and the positive definite property requirement stated above leaves much room for complex modeling taking into account the specificities of the material considered - anisotropy, in particular - and the effect of the porosity variations on this tensor, see e.g. [16] and references therein. We further point out that, considering that the privileged directions of the permeability tensor should follow the underlying deformations of the material principal directions, the form $\underline{\underline{K}}_{\mathrm{f}}$ is more intrinsic in nature than $\underline{\underline{k}}_{\mathrm{f}}$.

\subsection{Further modeling assumptions}

First, we suppose that the temperature is constant and uniform, as is e.g. well-justified when modeling living tissues. Furthermore, we emphasize that we have not - so far - made any particular assumption on the fluid state equation, namely, on the expression of $g_{m}(p, T)$. From now on, we will focus on incompressible fluid behavior, namely

$$
\rho_{\mathrm{f}}=\rho_{\mathrm{f} 0},
$$

which is not a restriction for most of the applications that we envision. In particular blood perfusion undoubtedly falls into that category, and it is also the case of pulmonary air flows given the range of applicable Reynolds number [3]. This directly entails

$$
g_{m}=\frac{p-p_{0}}{\rho_{\mathrm{f} 0}},
$$

where $p_{0}$ represents a reference pressure. Note that in this case we have

$$
\psi_{m}=g_{m}-\frac{p}{\rho_{\mathrm{f}}}=-\frac{p_{0}}{\rho_{\mathrm{f} 0}},
$$

hence $\psi_{m}$ is constant, which means that the fluid does not store any usable free energy, as can be expected with an incompressible behavior, indeed.

\subsection{Construction of an admissible Helmholtz free energy functional}

With the assumptions made on the fluid constitutive behavior and considering isothermal conditions, the Helmholtz free energy expression (40) reduces to

$$
\Psi=\Psi_{\mathrm{s}}\left(\underline{\underline{e}}, J_{\mathrm{s}}\right)-p_{0} J \phi,
$$

with $J_{\mathrm{s}}=J(1-\phi)$. Note that this makes $\Psi$ a function of $\underline{\underline{e}}$ and $m$ only, since $J_{\mathrm{s}}=J-J \phi$ and $J \phi=\frac{m}{\rho_{\mathrm{fo}}}+\phi_{0}$. This leads to the following constitutive equations providing the stress tensor in reversible transformations, namely, disregarding dissipation effects,

$$
\underline{\underline{\Sigma}}=\frac{\partial \Psi(\underline{\underline{e}}, m)}{\partial \underline{\underline{e}}}=\frac{\partial \Psi_{\mathrm{s}}}{\partial \underline{\underline{e}}}+\frac{\partial \Psi_{\mathrm{s}}}{\partial J_{\mathrm{s}}} \frac{\partial J}{\partial \underline{\underline{e}}}=\frac{\partial \Psi_{\mathrm{s}}}{\partial \underline{\underline{e}}}-p J \underline{\underline{C}}^{-1}
$$

with

$$
p=-\frac{\partial \Psi_{\mathrm{s}}}{\partial J_{\mathrm{s}}}
$$

as discussed in Remark 5. 
We are thus led to modeling the solid Helmholtz free energy $\Psi_{\mathrm{s}}$. Abundant work has already been devoted to this matter, although the literature is much more scarce on finite strain formulations, see e.g. [32, 17] and references therein. Here, our goal is not to give detailed formulations focused on specific types of porous materials, but rather to propose a general strategy inspired from hyperelastic concepts and well-adapted to computational purposes. The specific types of hyperelastic potentials to be selected will then depend on the materials considered in each application, and the corresponding constitutive parameters should be calibrated based on appropriate experimental trials, which the simplicity of the formulation should facilitate.

We propose to construct the free energy functional in the following - indeed, most natural - manner

$$
\Psi_{\mathrm{s}}=W^{\text {skel }}(\underline{\underline{e}})+W^{\text {bulk }}\left(J_{\mathrm{s}}\right),
$$

where $W^{\text {skel }}$ is a hyperelastic potential representing the constitutive behavior of the skeleton as a structure, while $W^{\text {bulk }}$ describes how the energy depends on the solid phase volume changes, against which the fluid interstitial pressure exerts mechanical work. For the bulk term, we can for example use the form classically employed in hyperelastic formulations, see e.g. [15, 35],

$$
W^{\text {bulk }}\left(J_{\mathrm{s}}\right)=\kappa\left(\frac{J_{\mathrm{s}}}{1-\phi_{0}}-1-\ln \left(\frac{J_{\mathrm{s}}}{1-\phi_{0}}\right)\right),
$$

with $\kappa$ a bulk modulus which can be chosen to be large in order to enforce nearly-incompressible behavior in the solid, hence total (approximate) incompressibility since the fluid was already assumed to be incompressible. We further emphasize that $W^{\text {skel }}$ should also incorporate some global bulk deformation energy, namely, provide a stress contribution which produces work on deformations associated with changes of volumes. In other words, we should have " $\partial W^{\text {skel }} / \partial J \neq$ 0 ". In fact, when considering an experiment in which the porous medium is "inflated" by increasing the fluid pressure while maintaining zero total stresses, this gives from (51)

$$
\frac{\partial W^{\text {skel }}}{\partial J}=\frac{\partial W^{\text {skel }}}{\partial \underline{\underline{e}}}: \frac{\underline{\underline{C}}}{3 J}=p
$$

which shows how this bulk energy provides resistance to internal pressure, as desired.

Note that the expression (53) gives a material which exactly satisfies the so-called "Terzaghi effective stress principle" - see e.g. [16] and references therein - since we then have

$$
\underline{\underline{\Sigma}}+p J \underline{\underline{C}}^{-1}=\frac{\partial W^{s k e l}}{\partial \underline{\underline{e}}}(\underline{\underline{e}})
$$

where the right-hand side only depends on the macroscopic strain tensor $\underline{\underline{e}}$, which means that the stress quantity $\underline{\underline{\underline{\Sigma}}}+p J \underline{\underline{C}}^{-1}$ entirely determines the (static) deformations of the skeleton. If we want to specifically characterize a porous material that departs from this principle, we can instead consider

$$
\Psi_{\mathrm{s}}=W^{\text {skel }}(\underline{\underline{e}})+W^{b u l k}\left(J_{\mathrm{s}} \frac{1-\phi_{0}}{\bar{J}_{\mathrm{S}}(J)}\right)
$$

where $\bar{J}_{\mathrm{s}}(J)$ is a function representing the variations of solid volume directly due to macroscopic volume changes in the absence of pore pressure, namely, the Poisson effect occurring "across the thickness" of the skeleton. For example, we could reasonably assume a linear ratio in the changes of volume, i.e.

$$
\frac{\bar{J}_{\mathrm{s}}(J)}{1-\phi_{0}}-1=\alpha(J-1) \quad \Leftrightarrow \quad \bar{J}_{\mathrm{s}}(J)=\left(1-\phi_{0}\right)(1-\alpha+\alpha J),
$$


where the ratio $\alpha$ should be less than one, and small when the compressibility of the solid material is low. Assuming (55), the stress tensor expression is now

$$
\underline{\underline{\Sigma}}=\frac{\partial W^{\text {skel }}}{\partial \underline{\underline{e}}}-p J \underline{\underline{C}}^{-1}-\left(W^{b u l k}\right)^{\prime} \frac{\left(1-\phi_{0}\right) J_{\mathrm{s}}\left(\bar{J}_{\mathrm{s}}\right)^{\prime}}{\left(\bar{J}_{\mathrm{s}}\right)^{2}} J \underline{\underline{C}}^{-1},
$$

where the last term shows that the formulation no longer satisfies the effective stress principle. An alternative expression can be obtained by incorporating (52), leading to

$$
\underline{\underline{\Sigma}}=\frac{\partial W^{\text {skel }}}{\partial \underline{\underline{e}}}-\left(1-\frac{J_{\mathrm{s}}\left(\bar{J}_{\mathrm{s}}\right)^{\prime}}{\bar{J}_{\mathrm{s}}}\right) p J \underline{\underline{C}}^{-1} .
$$

The Biot modulus $b$ being defined as the coefficient of the pressure term in the stress expression for infinitesimal transformations, we then have with the linear law (56) and upon linearization of the constitutive relation

$$
b=1-\alpha\left(1-\phi_{0}\right) .
$$

We also recall that the Biot modulus should tend to one when approaching solid incompressible behavior - namely, in which case we recover the effective stress principle - as is well-known and quite clear from our above discussion since $\alpha$ should vanish for incompressible materials. This justifies the use of the simpler construction (53) when considering nearly-incompressible behavior in the skeleton material.

Furthermore, as argued in [13], when large strains are considered special care must be exercised to maintain positive porosity. This can be achieved by including an additional penalization term in $\Psi_{\mathrm{s}}$, such as

$$
W^{\text {por }}=-\eta \ln \left(\frac{m}{\rho_{\mathrm{f} 0}}+\phi_{0}\right),
$$

which makes the fluid pressure tend to minus infinity through (52) when the porosity approaches zero, as is most natural. Accordingly, with this additional contribution the "inflated skeleton" equilibrium identity (54) is transformed into

$$
p=\frac{\partial W^{\text {skel }}}{\partial \underline{\underline{e}}}: \frac{\underline{\underline{C}}}{3 J}-\eta\left(\frac{m}{\rho_{\mathrm{f} 0}}+\phi_{0}\right)^{-1},
$$

which can also be considered for negative values of the pressure - namely, deflation.

Remark 6 - The additional porosity constraint $\phi \leq 1$ is naturally satisfied with the type of bulk potential proposed above, since $J_{\mathrm{s}}$ approaching zero then makes the free energy tend to infinity.

We point out that an alternative approach for formulating a poromechanics Helmholtz free energy functional is proposed and discussed in detail in [26] based on a decomposition of the strain tensor which, however, requires an isotropy assumption, see also [8] for related ideas. Our construction involves no such isotropy assumption and we - indeed - have among our chief objectives the modeling of living tissues, which are frequently made of highly anisotropic materials $[25,40]$. Moreover, in our approach, a natural extension can also be formulated to take into account an active contribution, in order to model muscle contraction - e.g. in the heart, see $[42,13,14]$ - which will be the subject of some further work.

Remark 7 -In this discussion, we have significantly departed from the approach taken in [13], in which the free energy functional was constructed by extending the linear theory of poromechanics. Note that the strategy proposed in the present paper is more general, and makes the enforcement of incompressible conditions more natural, in particular. 


\section{Governing equations and energy balance of the porous model}

In this section, we summarize and further analyze the governing equations of the complete poromechanics model constructed according to the guidelines discussed in the previous sections. We recall that we have obtained the following coupled system

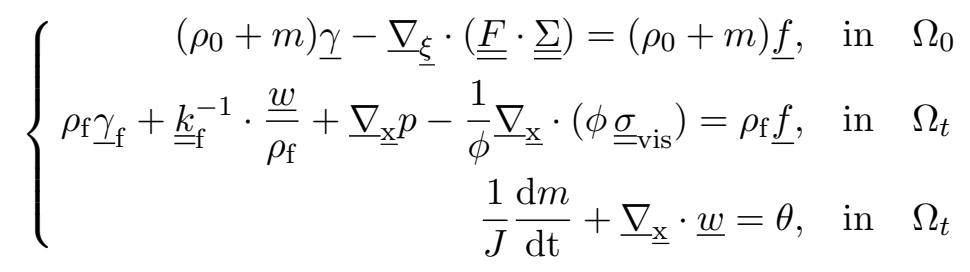

\subsection{Strong formulation}

As a first important remark, it is quite straightforward to see that we can transform the first equation of this system to express the conservation of momentum for the skeleton alone by subtracting the Lagrangian form of the second equation multiplied by $\phi$. This gives

$$
\rho_{\mathrm{s} 0}\left(1-\phi_{0}\right) \underline{\gamma}_{\mathrm{s}}-\underline{\nabla}_{\underline{\xi}} \cdot\left(\underline{\underline{F}} \cdot \underline{\underline{\Sigma}}_{\mathrm{s}}\right)-J \phi^{2} \underline{\underline{k}}_{\mathrm{f}}^{-1} \cdot\left(\underline{v}_{\mathrm{f}}-\underline{v}_{\mathrm{s}}\right)+p J \underline{\underline{F}}^{-T} \cdot \underline{\nabla}_{\underline{\xi}} \phi=\rho_{\mathrm{s} 0}\left(1-\phi_{0}\right) \underline{f},
$$

where $\underline{\underline{\mathrm{S}}}_{\mathrm{S}}$ denotes the skeleton contribution in the stress tensor, namely,

$$
\underline{\underline{\Sigma}}_{\mathrm{s}}=\underline{\underline{\Sigma}}-\phi \underline{\underline{\Sigma}}_{\mathrm{vis}}+\phi p J \underline{\underline{C}}^{-1} .
$$

Next, we proceed to reformulate the fluid inertia to make the second equation of System (58) explicit in the fluid velocity. We note that due to the conservation of fluid mass on an arbitrary subdomain $\Omega_{t}^{\prime}$,

$$
\frac{\mathrm{d}_{\mathrm{f}}}{\mathrm{dt}} \int_{\Omega_{t}^{\prime}} \rho_{\mathrm{f}} \phi d \Omega=\int_{\Omega_{t}^{\prime}} \theta d \Omega
$$

we have

$$
\int_{\Omega_{t}^{\prime}} \rho_{\mathrm{f}} \phi \underline{\gamma}_{\mathrm{f}} d \Omega=\frac{\mathrm{d}_{\mathrm{f}}}{\mathrm{dt}} \int_{\Omega_{t}^{\prime}} \rho_{\mathrm{f}} \phi \underline{v}_{\mathrm{f}} d \Omega-\int_{\Omega_{t}^{\prime}} \theta \underline{v}_{\mathrm{f}} d \Omega
$$

Using (5) we have

$$
\begin{aligned}
\frac{\mathrm{d}_{\mathrm{f}}}{\mathrm{dt}} \int_{\Omega_{t}^{\prime}} \rho_{\mathrm{f}} \phi \underline{v}_{\mathrm{f}} d \Omega & =\int_{\Omega_{t}^{\prime}}\left(\left.\frac{\partial}{\partial t}\left(\rho_{\mathrm{f}} \phi \underline{v}_{\mathrm{f}}\right)\right|_{\underline{\mathbf{x}}}+\underline{\nabla}_{\underline{\mathrm{x}}} \cdot\left(\rho_{\mathrm{f}} \phi \underline{v}_{\mathrm{f}} \otimes \underline{v}_{\mathrm{f}}\right)\right) d \Omega \\
& =\int_{\Omega_{t}^{\prime}}\left(\left.\frac{\partial}{\partial t}\left(\rho_{\mathrm{f}} \phi \underline{v}_{\mathrm{f}}\right)\right|_{\underline{\mathbf{x}}}+\underline{\nabla}_{\underline{\mathrm{x}}} \cdot\left(\rho_{\mathrm{f}} \phi \underline{v}_{\mathrm{f}} \otimes \underline{v}_{\mathrm{s}}\right)\right) d \Omega+\int_{\Omega_{t}^{\prime}} \underline{\nabla}_{\underline{\mathrm{x}}} \cdot\left(\rho_{\mathrm{f}} \phi \underline{v}_{\mathrm{f}} \otimes\left(\underline{v}_{\mathrm{f}}-\underline{v}_{\mathrm{s}}\right)\right) d \Omega \\
& =\frac{\mathrm{d}}{\mathrm{dt}}\left(\int_{\Omega_{t}^{\prime}} \rho_{\mathrm{f}} \phi \underline{v}_{\mathrm{f}} d \Omega\right)+\int_{\Omega_{t}^{\prime}} \underline{\nabla}_{\underline{\mathrm{x}}} \cdot\left(\rho_{\mathrm{f}} \phi \underline{v}_{\mathrm{f}} \otimes\left(\underline{v}_{\mathrm{f}}-\underline{v}_{\mathrm{s}}\right)\right) d \Omega \\
& =\frac{\mathrm{d}}{\mathrm{dt}}\left(\int_{\Omega_{0}^{\prime}} J \rho_{\mathrm{f}} \phi \underline{v}_{\mathrm{f}} d \Omega\right)+\int_{\Omega_{t}^{\prime}} \underline{\nabla}_{\underline{\mathrm{x}}} \cdot\left(\rho_{\mathrm{f}} \phi \underline{v}_{\mathrm{f}} \otimes\left(\underline{v}_{\mathrm{f}}-\underline{v}_{\mathrm{s}}\right)\right) d \Omega \\
& =\left.\int_{\Omega_{0}^{\prime}} \frac{\partial}{\partial t}\left(J \rho_{\mathrm{f}} \phi \underline{v}_{\mathrm{f}}\right)\right|_{\underline{\xi}} d \Omega+\int_{\Omega_{t}^{\prime}} \underline{\nabla}_{\underline{\mathrm{x}}} \cdot\left(\rho_{\mathrm{f}} \phi \underline{v}_{\mathrm{f}} \otimes\left(\underline{v}_{\mathrm{f}}-\underline{v}_{\mathrm{s}}\right)\right) d \Omega
\end{aligned}
$$

which provides the identity

$$
\rho_{\mathrm{f}} \phi \underline{\gamma}_{\mathrm{f}}=\frac{1}{J} \frac{\mathrm{d}}{\mathrm{dt}}\left(J \rho_{\mathrm{f}} \phi \underline{v}_{\mathrm{f}}\right)+\underline{\nabla}_{\underline{\mathrm{x}}} \cdot\left(\rho_{\mathrm{f}} \phi \underline{v}_{\mathrm{f}} \otimes\left(\underline{v}_{\mathrm{f}}-\underline{v}_{\mathrm{s}}\right)\right)-\theta \underline{v}_{\mathrm{f}} .
$$

Gathering the above equations, we thus obtain the following summary. 


\section{System of porous medium equations - Strong formulation}

$$
\left\{\begin{aligned}
& \rho_{\mathrm{s} 0}\left(1-\phi_{0}\right) \frac{\mathrm{d} \underline{v}_{\mathrm{s}}}{\mathrm{dt}}-\underline{\nabla}_{\underline{\xi}} \cdot\left(\underline{\underline{F}} \cdot \underline{\underline{\Sigma}}_{\mathrm{s}}\right)-J \phi^{2} \underline{\underline{k}}_{\mathrm{f}}^{-1} \cdot\left(\underline{v}_{\mathrm{f}}-\underline{v}_{\mathrm{s}}\right) \\
&+p J \underline{\underline{F}}^{-T} \cdot \underline{\nabla}_{\underline{\xi}} \phi=\rho_{\mathrm{s} 0}\left(1-\phi_{0}\right) \underline{f}, \quad \text { in } \quad \Omega_{0} \\
& \frac{1}{J} \frac{\mathrm{d}}{\mathrm{dt}}\left(J \rho_{\mathrm{f}} \phi \underline{v}_{\mathrm{f}}\right)+\underline{\nabla}_{\underline{\mathrm{x}}} \cdot\left(\rho_{\mathrm{f}} \phi \underline{v}_{\mathrm{f}} \otimes\left(\underline{v}_{\mathrm{f}}-\underline{v}_{\mathrm{s}}\right)\right)-\theta \underline{v}_{\mathrm{f}}+\phi^{2} \underline{k}_{\mathrm{f}}^{-1} \cdot\left(\underline{v}_{\mathrm{f}}-\underline{v}_{\mathrm{s}}\right) \\
&+\phi \underline{\nabla}_{\underline{\mathrm{x}}} p-\underline{\nabla}_{\underline{\mathrm{x}}} \cdot\left(\phi \underline{\sigma}_{\mathrm{vis}}\right)=\rho_{\mathrm{f}} \phi \underline{f}, \quad \text { in } \quad \Omega_{t} \\
& \frac{1}{J} \frac{\mathrm{d}}{\mathrm{dt}}\left(J \rho_{\mathrm{f}} \phi\right)+\underline{\nabla}_{\underline{\mathrm{x}}} \cdot\left(\rho_{\mathrm{f}} \phi\left(\underline{v}_{\mathrm{f}}-\underline{v}_{\mathrm{s}}\right)\right)=\theta, \quad \text { in } \quad \Omega_{t}
\end{aligned}\right.
$$

In this system, the first two equations can be seen as governing the kinematical variables of the two phases - namely, the solid displacement and the fluid velocity, respectively - while the third equation is here written with the porosity as a primary variable, albeit could be seen as equivalently bearing on the fluid mass by (16), or on the pressure which can be eliminated for by using (52). Some special caution should be exercized concerning the boundary conditions to be jointly prescribed on the kinematical variables, the most common combinations being as follows:

- Dirichlet boundary conditions for both phases, namely, prescribed skeleton displacements and fluid velocities:

$$
\underline{y}=\underline{y}^{\mathrm{pr}}, \quad \underline{v}_{\mathrm{f}}=\underline{v}_{\mathrm{f}}^{\mathrm{pr}},
$$

on the subpart of the boundary which we denote by $\Gamma_{0}^{D}$ in the reference configuration and $\Gamma_{t}^{D}$ in the current configuration;

- Neumann boundary conditions - namely, prescribed forces - for both phases together, with proportional repartition of boundary traction:

$$
\underline{\underline{\sigma}} \cdot \underline{n}=\underline{t} \Leftrightarrow \underline{\underline{F}} \cdot \underline{\underline{\Sigma}} \cdot \underline{n}_{0}=\underline{t}_{0}, \quad \underline{\underline{\sigma}} \cdot \underline{n}=\underline{t},
$$

on $\Gamma_{0}^{N}\left(\right.$ or $\left.\Gamma_{t}^{N}\right)$;

- Neumann boundary condition for the global mixture, but vanishing fluid flux and proportional repartition of tangential boundary traction:

$$
\underline{\underline{\sigma}} \cdot \underline{n}=\underline{t} \Leftrightarrow \underline{\underline{F}} \cdot \underline{\underline{\Sigma}} \cdot \underline{n}_{0}=\underline{t}_{0}, \quad \Pi_{\tau}\left(\underline{\underline{\sigma}}_{\mathrm{f}} \cdot \underline{n}\right)=\Pi_{\tau}(\underline{t}), \quad\left(\underline{v}_{\mathrm{f}}-\underline{v}_{\mathrm{s}}\right) \cdot \underline{n}=0,
$$

on $\Gamma_{0}^{N \text { nof }}$ (or $\Gamma_{t}^{N \text { nof }}$ ), where $\Pi_{\tau}=\underline{\underline{\mathbb{1}}}-\underline{n} \otimes \underline{n}$ denotes the projection onto the tangential plane;

- Neumann boundary condition for the global mixture with fluid velocity coinciding with the solid velocity (no sliding):

$$
\underline{\underline{\sigma}} \cdot \underline{n}=\underline{t} \Leftrightarrow \underline{\underline{F}} \cdot \underline{\underline{\Sigma}} \cdot \underline{n}_{0}=\underline{t}_{0}, \quad \underline{v}_{\mathrm{f}}=\underline{v}_{\mathrm{s}}
$$

on $\Gamma_{0}^{N \operatorname{nos}}\left(\right.$ or $\left.\Gamma_{t}^{N \text { nos }}\right)$.

Remark 8 - We recognize in $(60)_{2}$ an equation very similar to the conservative form of the so-called Arbitrary Lagrangian Eulerian (ALE) formulation of the compressible Navier-Stokes equations $[24,36]$, where here the domain velocity is given by the skeleton physical velocity and the product $\rho_{\mathrm{f}} \phi$ plays the role of a varying fluid mass density. However, compared with standard fluid-structure interaction problems, we have the additional distributed coupling term $\underline{k}_{\mathrm{f}}^{-1} \cdot\left(\underline{v}_{\mathrm{f}}-\underline{v}_{\mathrm{s}}\right)$ representing the interaction between the two phases. 


\subsection{Weak formulation}

From the above system written in strong form, we can obtain an equivalent weak form - in other words, a variational formulation - by multiplying each equation by a corresponding test function. This gives the following system.

\section{System of porous medium equations - Weak formulation}

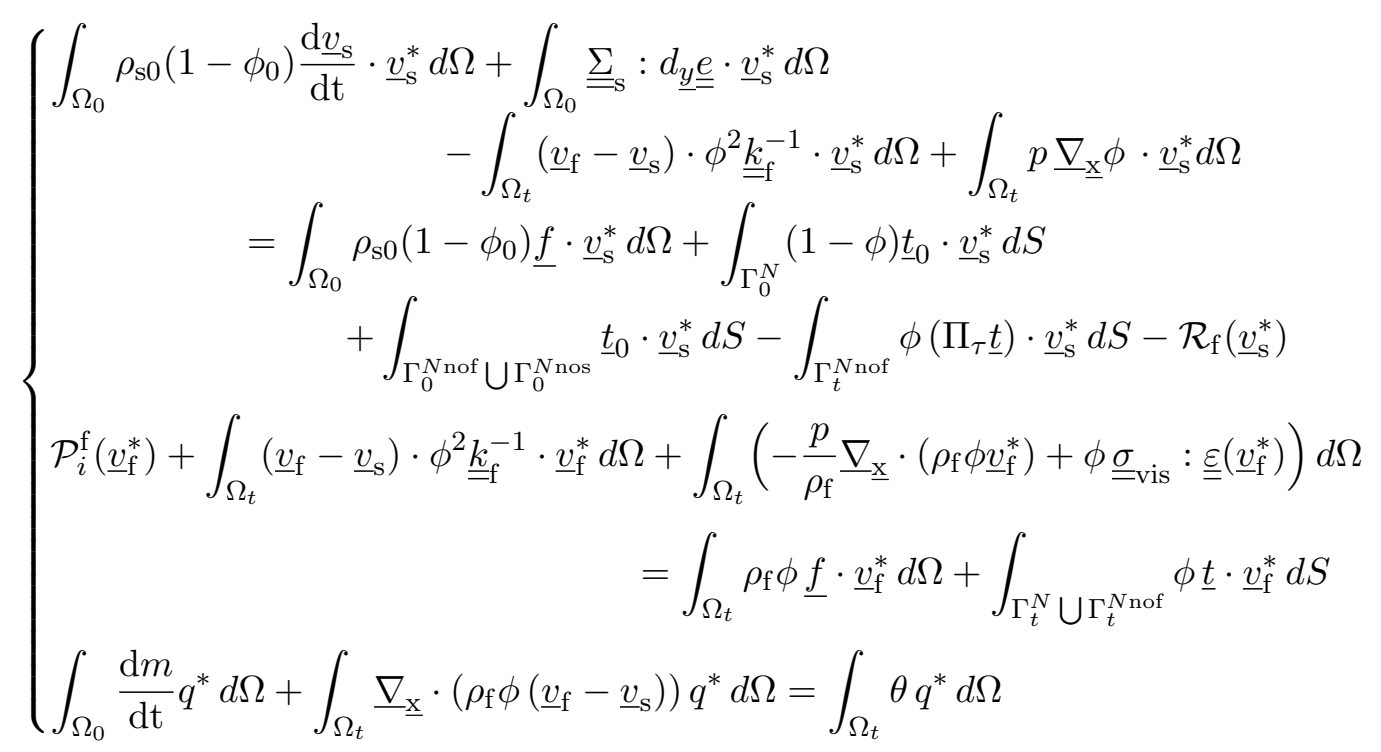

to be verified for any admissible test functions $\underline{v}_{\mathrm{s}}^{*}, \underline{v}_{\mathrm{f}}^{*}$ and $q^{*}$, and with the virtual power of fluid inertia forces given by

$$
\mathcal{P}_{i}^{\mathrm{f}}\left(\underline{v}_{\mathrm{f}}^{*}\right)=\int_{\Omega_{0}} \frac{\mathrm{d}}{\mathrm{dt}}\left(J \rho_{\mathrm{f}} \phi \underline{v}_{\mathrm{f}}\right) \cdot \underline{v}_{\mathrm{f}}^{*} d \Omega+\int_{\Omega_{t}} \underline{\nabla}_{\underline{\mathrm{x}}} \cdot\left(\rho_{\mathrm{f}} \phi \underline{v}_{\mathrm{f}} \otimes\left(\underline{v}_{\mathrm{f}}-\underline{v}_{\mathrm{s}}\right)\right) \cdot \underline{v}_{\mathrm{f}}^{*} d \Omega-\int_{\Omega_{t}} \theta \underline{v}_{\mathrm{f}} \cdot \underline{v}_{\mathrm{f}}^{*} d \Omega,
$$

while

$$
\begin{aligned}
\mathcal{R}_{\mathrm{f}}\left(\underline{v}_{\mathrm{s}}^{*}\right)= & \int_{\Gamma_{t}^{N \text { nos }}} \phi\left(\underline{\underline{\sigma}}_{\mathrm{f}} \cdot \underline{n}\right) \cdot \underline{v}_{\mathrm{s}}^{*} d S+\int_{\Gamma_{t}^{N \text { nof }}} \phi\left(\underline{\underline{\sigma}}_{\mathrm{f}} \cdot \underline{n}\right) \cdot \underline{n}\left(\underline{v}_{\mathrm{s}}^{*} \cdot \underline{n}\right) d S \\
= & \mathcal{P}_{i}^{\mathrm{f}}\left(\underline{v}_{\mathrm{s}}^{*}\right)+\int_{\Omega_{t}}\left(\underline{v}_{\mathrm{f}}-\underline{v}_{\mathrm{s}}\right) \cdot \phi^{2} \underline{\underline{k}}_{\mathrm{f}}^{-1} \cdot \underline{v}_{\mathrm{s}}^{*} d \Omega \\
& +\int_{\Omega_{t}}\left(-\frac{\underline{p}}{\rho_{\mathrm{f}}} \underline{\nabla}_{\underline{\mathrm{x}}} \cdot\left(\rho_{\mathrm{f}} \phi \underline{v}_{\mathrm{s}}^{*}\right)+\phi \underline{\sigma} \underline{\mathrm{vis}}: \underline{\varepsilon}\left(\underline{v}_{\mathrm{s}}^{*}\right)\right) d \Omega \\
& -\int_{\Omega_{t}} \rho_{\mathrm{f}} \phi \underline{f} \cdot \underline{v}_{\mathrm{s}}^{*} d \Omega-\int_{\Gamma_{t}^{N}} \phi \underline{t} \cdot \underline{v}_{\mathrm{s}}^{*} d S-\int_{\Gamma_{t}^{N \text { nof }}} \phi\left(\Pi_{\tau} \underline{t}\right) \cdot \underline{v}_{\mathrm{s}}^{*} d S
\end{aligned}
$$

is the residual of the second equation (namely, the virtual work of fluid reaction forces) with respect to the fluid-related Dirichlet boundary conditions prescribed on the boundaries $\Gamma_{t}^{N \text { nos }}$ and $\Gamma_{t}^{N \text { nof }}$. The complete Dirichlet boundary conditions for this system are

$$
\left\{\begin{array}{l}
\underline{y}^{=} \underline{y}^{\mathrm{pr}}, \quad \underline{v}_{\mathrm{f}}=\underline{v}_{\mathrm{f}}^{\mathrm{pr}}, \text { on } \Gamma_{0}^{D} \\
\underline{v}_{\mathrm{f}} \cdot \underline{n}=\underline{v}_{\mathrm{s}} \cdot \underline{n}, \text { on } \Gamma_{t}^{N \text { nof }} \\
\underline{v}_{\mathrm{f}}=\underline{v}_{\mathrm{s}}, \text { on } \Gamma_{t}^{N \text { nos }}
\end{array}\right.
$$

and the respective test functions must accordingly satisfy the corresponding homogeneous conditions. Note that in this variational formulation we have used a combination of Lagrangian 
and Eulerian terms according to which is the most effective form for computational purposes. In addition, the conservation of mass equation is now written with the added mass variable $m$, which is quite natural since the Helmholtz free energy functional is a function of this variable.

\subsection{Variants of equations}

Of course, we have an equivalent system when substituting $(61)_{1}$ with the total conservation of momentum in a weak form - namely, we simply substitute the expression of the residual term $\mathcal{R}_{\mathrm{f}}\left(\underline{v}_{\mathrm{s}}^{*}\right)$ - which gives the following alternative formulation.

System of porous medium equations - Second weak formulation

$$
\left\{\begin{array}{l}
\int_{\Omega_{0}} \rho_{\mathrm{s} 0}\left(1-\phi_{0}\right) \frac{\mathrm{d} \underline{\underline{v}}}{\mathrm{dt}} \cdot \underline{v}_{\mathrm{s}}^{*} d \Omega+\mathcal{P}_{i}^{\mathrm{f}}\left(\underline{v}_{\mathrm{s}}^{*}\right)+\int_{\Omega_{0}} \underline{\underline{\Sigma}}: d_{\underline{y}} \underline{\underline{e}} \cdot \underline{v}_{\mathrm{s}}^{*} d \Omega \\
\quad=\int_{\Omega_{0}}\left(\rho_{0}+m\right) \underline{f} \cdot \underline{v}_{\mathrm{s}}^{*} d \Omega+\int_{\Gamma_{0}^{N} \cup \Gamma_{0}^{N \text { nof }} \cup \Gamma_{0}^{N \text { nos }}} \underline{t}_{0} \cdot \underline{v}_{\mathrm{s}}^{*} d S \\
\text { Same as }(61)_{2} \\
\text { Same as }(61)_{3}
\end{array}\right.
$$

Note that this form substantially simplifies the expression of the virtual work of external forces.

Furthermore, we can obtain alternative expressions of the fluid inertia power, as is usually done in standard fluid-structure interaction formulations. For example, the form employed in [35] is obtained by noting that

$$
\left.\frac{\partial}{\partial t}\left(J \rho_{\mathrm{f}} \phi \underline{v}_{\mathrm{f}}\right)\right|_{\underline{\xi}}=\left.\frac{\partial}{\partial t}\left(J \rho_{\mathrm{f}} \phi\right)\right|_{\underline{\xi}} \underline{v}_{\mathrm{f}}+\left.J \rho_{\mathrm{f}} \phi \frac{\partial \underline{v}_{\mathrm{f}}}{\partial t}\right|_{\underline{\xi}}
$$

while

$$
\left.J \rho_{\mathrm{f}} \phi \frac{\partial \underline{v}_{\mathrm{f}}}{\partial t}\right|_{\underline{\xi}}=\sqrt{J \rho_{\mathrm{f}} \phi}\left(\left.\frac{\partial}{\partial t}\left(\sqrt{J \rho_{\mathrm{f}} \phi} \underline{v}_{\mathrm{f}}\right)\right|_{\underline{\xi}}-\left.\frac{1}{2 \sqrt{J \rho_{\mathrm{f}} \phi}} \frac{\partial}{\partial t}\left(J \rho_{\mathrm{f}} \phi\right)\right|_{\underline{\xi}_{\mathrm{f}}}\right) .
$$

We then infer

$$
\begin{aligned}
\left.\frac{\partial}{\partial t}\left(J \rho_{\mathrm{f}} \phi \underline{v}_{\mathrm{f}}\right)\right|_{\underline{\xi}} & =\left.\sqrt{J \rho_{\mathrm{f}} \phi} \frac{\partial}{\partial t}\left(\sqrt{J \rho_{\mathrm{f}} \phi} \underline{v}_{\mathrm{f}}\right)\right|_{\underline{\xi}}+\left.\frac{1}{2} \frac{\partial}{\partial t}\left(J \rho_{\mathrm{f}} \phi\right)\right|_{\underline{\xi}_{\mathrm{f}}} \\
& =\left.\sqrt{J \rho_{\mathrm{f}} \phi} \frac{\partial}{\partial t}\left(\sqrt{J \rho_{\mathrm{f}} \phi} \underline{v}_{\mathrm{f}}\right)\right|_{\underline{\xi}}+\frac{J}{2}\left(\theta-\underline{\nabla}_{\underline{\mathrm{x}}} \cdot\left(\rho_{\mathrm{f}} \phi\left(\underline{v}_{\mathrm{f}}-\underline{v}_{\mathrm{s}}\right)\right) \underline{v}_{\mathrm{f}},\right.
\end{aligned}
$$

where we have used (16) and the Eulerian form of (17) in the last substitution. Hence, we can transform (59) into the second fluid inertia identity

$$
\rho_{\mathrm{f}} \phi \underline{\gamma}_{\mathrm{f}}=\frac{1}{J} \sqrt{J \rho_{\mathrm{f}} \phi} \frac{\mathrm{d}}{\mathrm{dt}}\left(\sqrt{J \rho_{\mathrm{f}} \phi} \underline{v}_{\mathrm{f}}\right)-\frac{1}{2} \underline{\nabla}_{\underline{\mathrm{x}}} \cdot\left(\rho_{\mathrm{f}} \phi\left(\underline{v}_{\mathrm{f}}-\underline{v}_{\mathrm{s}}\right)\right) \underline{v}_{\mathrm{f}}+\underline{\nabla}_{\underline{\mathrm{x}}} \cdot\left(\rho_{\mathrm{f}} \phi \underline{v}_{\mathrm{f}} \otimes\left(\underline{v}_{\mathrm{f}}-\underline{v}_{\mathrm{s}}\right)\right)-\frac{1}{2} \theta \underline{v}_{\mathrm{f}},
$$

or equivalently in a weak form

$$
\begin{aligned}
\mathcal{P}_{i}^{\mathrm{f}}\left(\underline{v}^{*}\right)=\int_{\Omega_{0}} \frac{\mathrm{d}}{\mathrm{dt}}\left(\sqrt{J \rho_{\mathrm{f}} \phi} \underline{v}_{\mathrm{f}}\right) \cdot & \sqrt{J \rho_{\mathrm{f}} \phi} \underline{v}^{*} d \Omega-\frac{1}{2} \int_{\Omega_{t}} \underline{\nabla}_{\underline{\mathrm{x}}} \cdot\left(\rho_{\mathrm{f}} \phi\left(\underline{v}_{\mathrm{f}}-\underline{v}_{\mathrm{s}}\right)\right) \underline{v}_{\mathrm{f}} \cdot \underline{v}^{*} d \Omega \\
& +\int_{\Omega_{t}} \underline{\nabla}_{\underline{\mathrm{x}}} \cdot\left(\rho_{\mathrm{f}} \phi \underline{v}_{\mathrm{f}} \otimes\left(\underline{v}_{\mathrm{f}}-\underline{v}_{\mathrm{s}}\right)\right) \cdot \underline{v}^{*} d \Omega-\frac{1}{2} \int_{\Omega_{t}} \theta \underline{v}_{\mathrm{f}} \cdot \underline{v}^{*} d \Omega .
\end{aligned}
$$

As we will see in the next section, this form is particularly useful when dealing with energy balances. Hence, we also expect this expression to be instrumental when devising energyconsistent discretization schemes, as exemplified in Section 7. 


\subsection{Energy balance}

We now make use of the above-derived system equations to reformulate directly the energy principle for the porous medium, in a form providing explicit estimates for the solution of the system (60) - or the associated weak forms (61)-(63). This type of estimate is crucial in particular for analyzing the mathematical properties of the solutions and their discretizations. Defining the total Helmholtz free energy

$$
\mathcal{W}=\int_{\Omega_{0}} \Psi d \Omega
$$

we have the following result.

\section{Theorem 7}

The solution of the general poromechanics problem written in (60) and (61)-(63) in strong and weak forms, respectively, satisfies the following energy balance

$$
\begin{array}{r}
\frac{\mathrm{d} \mathcal{K}}{\mathrm{dt}}+\frac{\mathrm{d} \mathcal{W}}{\mathrm{dt}}=-\int_{\Omega_{0}} \frac{\partial \Psi_{\mathrm{damp}}}{\partial \underline{\underline{\dot{e}}}}: \underline{\underline{e}} d \Omega-\int_{\Omega_{t}} \phi \underline{\underline{\sigma}}_{v i s}: \underline{\underline{\varepsilon}}\left(\underline{v}_{\mathrm{f}}\right) d \Omega-\int_{\Omega_{t}}\left(\underline{v}_{\mathrm{f}}-\underline{v}_{\mathrm{s}}\right) \cdot \phi^{2} \underline{\underline{k}}_{\mathrm{f}}^{-1} \cdot\left(\underline{v}_{\mathrm{f}}-\underline{v}_{\mathrm{s}}\right) d \Omega \\
+\mathcal{P}_{\text {ext }}^{\text {total }}+\mathcal{J}_{\mathcal{K} b}+\mathcal{J}_{\mathcal{K} \theta}+\mathcal{J}_{\mathcal{W} b}+\mathcal{J}_{\mathcal{G} \theta},
\end{array}
$$

with

$$
\mathcal{J}_{\mathcal{K} b}=-\frac{1}{2} \int_{\partial \Omega_{t}} \rho_{\mathrm{f}} \phi \underline{v}_{\mathrm{f}}^{2}\left(\underline{v}_{\mathrm{f}}-\underline{v}_{\mathrm{s}}\right) \cdot \underline{n} d S, \quad \mathcal{J}_{\mathcal{K} \theta}=\frac{1}{2} \int_{\Omega_{t}} \underline{v}_{\mathrm{f}}^{2} \theta d \Omega,
$$

the incoming rates of fluid kinetic energy due to the boundary flow and source term, respectively, and

$$
\mathcal{J}_{\mathcal{W} b}=-\int_{\partial \Omega_{t}} \rho_{\mathrm{f}} \phi \psi_{m}\left(\underline{v}_{\mathrm{f}}-\underline{v}_{\mathrm{s}}\right) \cdot \underline{n} d S, \quad \mathcal{J}_{\mathcal{G} \theta}=\int_{\Omega_{t}} g_{m} \theta d \Omega,
$$

similar incoming rates of Helmholtz and Gibbs free energies.

Proof. First we consider the fluid inertia power in the form (65) applied to the fluid velocity. We start with the following identities

$$
\underline{\nabla}_{\underline{\mathrm{x}}} \cdot\left(\rho_{\mathrm{f}} \phi \underline{v}_{\mathrm{f}}^{2}\left(\underline{v}_{\mathrm{f}}-\underline{v}_{\mathrm{s}}\right)\right)=\underline{v}_{\mathrm{f}}^{2} \underline{\nabla}_{\underline{\mathrm{x}}} \cdot\left(\rho_{\mathrm{f}} \phi\left(\underline{v}_{\mathrm{f}}-\underline{v}_{\mathrm{s}}\right)\right)+2\left(\underline{v}_{\mathrm{f}} \cdot \underline{\underline{\nabla}}_{\underline{\mathrm{x}}} \underline{v}_{\mathrm{f}}\right) \rho_{\mathrm{f}} \phi\left(\underline{v}_{\mathrm{f}}-\underline{v}_{\mathrm{s}}\right),
$$

and

$$
\underline{\nabla}_{\underline{\mathrm{x}}} \cdot\left(\rho_{\mathrm{f}} \phi \underline{v}_{\mathrm{f}} \otimes\left(\underline{v}_{\mathrm{f}}-\underline{v}_{\mathrm{s}}\right)\right) \cdot \underline{v}_{\mathrm{f}}=\underline{v}_{\mathrm{f}}^{2} \underline{\nabla}_{\mathrm{x}} \cdot\left(\rho_{\mathrm{f}} \phi\left(\underline{v}_{\mathrm{f}}-\underline{v}_{\mathrm{s}}\right)\right)+\left(\underline{v}_{\mathrm{f}} \cdot \underline{\nabla}_{\underline{\mathrm{x}}} \underline{v}_{\mathrm{f}}\right) \rho_{\mathrm{f}} \phi\left(\underline{v}_{\mathrm{f}}-\underline{v}_{\mathrm{s}}\right) .
$$

Hence,

$$
\underline{\nabla}_{\underline{\mathrm{x}}} \cdot\left(\rho_{\mathrm{f}} \phi \underline{v}_{\mathrm{f}} \otimes\left(\underline{v}_{\mathrm{f}}-\underline{v}_{\mathrm{s}}\right)\right) \cdot \underline{v}_{\mathrm{f}}=\frac{1}{2} \underline{v}_{\mathrm{f}}^{2} \underline{\nabla}_{\underline{\mathrm{x}}} \cdot\left(\rho_{\mathrm{f}} \phi\left(\underline{v}_{\mathrm{f}}-\underline{v}_{\mathrm{s}}\right)\right)+\frac{1}{2} \underline{\nabla}_{\underline{\mathrm{x}}} \cdot\left(\rho_{\mathrm{f}} \phi \underline{v}_{\mathrm{f}}^{2}\left(\underline{v}_{\mathrm{f}}-\underline{v}_{\mathrm{s}}\right)\right),
$$

which gives

$$
\begin{aligned}
\mathcal{P}_{i}^{\mathrm{f}}\left(\underline{v}_{\mathrm{f}}\right) & =\int_{\Omega_{0}} \frac{\mathrm{d}}{\mathrm{dt}}\left(\sqrt{J \rho_{\mathrm{f}} \phi} \underline{v}_{\mathrm{f}}\right) \cdot \sqrt{J \rho_{\mathrm{f}} \phi} \underline{v}_{\mathrm{f}} d \Omega+\int_{\Omega_{t}} \frac{1}{2} \underline{\nabla}_{\underline{\mathrm{x}}} \cdot\left(\rho_{\mathrm{f}} \phi \underline{v}_{\mathrm{f}}^{2}\left(\underline{v}_{\mathrm{f}}-\underline{v}_{\mathrm{s}}\right)\right) d \Omega-\frac{1}{2} \int_{\Omega_{t}} \theta \underline{v}_{\mathrm{f}}^{2} d \Omega \\
& =\frac{\mathrm{d} \mathcal{K}_{\mathrm{f}}}{\mathrm{dt}}+\int_{\partial \Omega_{t}} \frac{1}{2} \rho_{\mathrm{f}} \phi \underline{v}_{\mathrm{f}}^{2}\left(\underline{v}_{\mathrm{f}}-\underline{v}_{\mathrm{s}}\right) \cdot \underline{n} d S-\frac{1}{2} \int_{\Omega_{t}} \theta \underline{v}_{\mathrm{f}}^{2} d \Omega \\
& =\frac{\mathrm{d} \mathcal{K}_{\mathrm{f}}}{\mathrm{dt}}-\mathcal{J}_{\mathcal{K} b}-\mathcal{J}_{\mathcal{K} \theta} .
\end{aligned}
$$


Therefore, adding the weak form $(63)_{1}$ tested with $\underline{v}_{\mathrm{S}}$ to the weak form $(61)_{2}$ tested with $\left(\underline{v}_{\mathrm{f}}-\underline{v}_{\mathrm{S}}\right)$ we obtain

$$
\begin{aligned}
\frac{\mathrm{d} \mathcal{K}_{\mathrm{s}}}{\mathrm{dt}} & +\frac{\mathrm{d} \mathcal{K}_{\mathrm{f}}}{\mathrm{dt}}+\int_{\Omega_{0}} \frac{\partial \Psi}{\partial \underline{\underline{e}}}: d_{\underline{y}} \underline{\underline{e}} \cdot \underline{v}_{\mathrm{s}} d \Omega+\int_{\Omega_{t}}\left(-\frac{p}{\rho_{\mathrm{f}}} \underline{\nabla}_{\underline{\mathrm{x}}} \cdot\left(\rho_{\mathrm{f}} \phi\left(\underline{v}_{\mathrm{f}}-\underline{v}_{\mathrm{s}}\right)\right)+\phi \underline{\underline{\sigma}}_{\mathrm{vis}}: \underline{\underline{\varepsilon}}\left(\underline{v}_{\mathrm{f}}\right)\right) d \Omega \\
= & -\int_{\Omega_{0}} \frac{\partial \Psi_{\mathrm{damp}}}{\partial \underline{\underline{e}}}: d_{\underline{y}} \underline{\underline{e}} \cdot \underline{v}_{\mathrm{s}} d \Omega-\int_{\Omega_{t}}\left(\underline{v}_{\mathrm{f}}-\underline{v}_{\mathrm{s}}\right) \cdot \phi^{2} \underline{\underline{k}}_{\mathrm{f}}^{-1} \cdot\left(\underline{v}_{\mathrm{f}}-\underline{v}_{\mathrm{s}}\right) d \Omega+\mathcal{P}_{\mathrm{ext}}^{\text {total }}+\mathcal{J}_{\mathcal{K} b}+\mathcal{J}_{\mathcal{K} \theta} .
\end{aligned}
$$

Note that this identity also holds when prescribing non-homogeneous Dirichlet boundary conditions - even though the test functions in the variational system are assumed to satisfy homogeneous conditions - since then the remaining residuals are exactly compensated by adequate terms in the power of external forces, by definition of these residuals. Finally, testing $(61)_{3}$ with $\frac{p-p_{0}}{\rho_{\mathrm{f}}}$ we have

$$
\int_{\Omega_{t}} \frac{p-p_{0}}{\rho_{\mathrm{f}}} \underline{\nabla}_{\underline{\mathrm{x}}} \cdot\left(\rho_{\mathrm{f}} \phi\left(\underline{v}_{\mathrm{f}}-\underline{v}_{\mathrm{s}}\right)\right) d \Omega+\int_{\Omega_{0}} \frac{p-p_{0}}{\rho_{\mathrm{f}}} \frac{\mathrm{d} m}{\mathrm{dt}} d \Omega=\int_{\Omega_{t}} \frac{p-p_{0}}{\rho_{\mathrm{f}}} \theta d \Omega,
$$

which by the Stokes formula gives

$$
\begin{aligned}
-\int_{\Omega_{t}} \frac{p}{\rho_{\mathrm{f}}} \underline{\nabla}_{\underline{\mathrm{x}}} \cdot\left(\rho_{\mathrm{f}} \phi\left(\underline{v}_{\mathrm{f}}-\underline{v}_{\mathrm{s}}\right)\right) d \Omega & =\int_{\Omega_{0}} \frac{p-p_{0}}{\rho_{\mathrm{f}}} \frac{\mathrm{d} m}{\mathrm{dt}} d \Omega-\int_{\partial \Omega_{t}} \rho_{\mathrm{f}} \phi \frac{p_{0}}{\rho_{\mathrm{f}}}\left(\underline{v}_{\mathrm{f}}-\underline{v}_{\mathrm{s}}\right) \cdot \underline{n} d S \\
& =\int_{\Omega_{0}} g_{m} \frac{\mathrm{d} m}{\mathrm{dt}} d \Omega-\mathcal{J}_{\mathcal{W} b}-\mathcal{J}_{\mathcal{G} \theta} .
\end{aligned}
$$

Therefore, using $\underline{\underline{\dot{e}}}=d_{\underline{y}} \underline{\underline{e}} \cdot \underline{v}_{\mathrm{s}}$, we have

$$
\int_{\Omega_{0}}\left(\frac{\partial \Psi}{\partial \underline{\underline{e}}}: d_{\underline{y}} \underline{\underline{e}} \cdot \underline{v}_{\mathrm{s}}+g_{m} \frac{\mathrm{d} m}{\mathrm{dt}}\right) d \Omega=\int_{\Omega_{0}}\left(\frac{\partial \Psi}{\partial \underline{\underline{e}}}: \underline{\underline{e}}+\frac{\partial \Psi}{\partial m} \dot{m}\right) d \Omega=\frac{\mathrm{d} \mathcal{W}}{\mathrm{dt}},
$$

which concludes the proof.

In Theorem 7, the first three contributions appearing in the right-hand side of the balance (66) are negative and respectively correspond to dissipation effects in the solid, in the fluid and in the interaction between the two components. The last four contributions correspond to source terms and external forces, hence they vanish for an isolated system.

Remark 9 - An incoming rate of Gibbs free energy can be naturally interpreted as the sum of external power performed on the corresponding added mass and increase of fluid Helmholtz free energy associated with this mass, as e.g.

$$
\mathcal{J}_{\mathcal{G} \theta}=\int_{\Omega_{t}}\left(p \frac{\theta}{\rho_{\mathrm{f}}}+\psi_{m} \theta\right) d \Omega
$$

The reason why it is the Helmholtz free energy which appears in $\mathcal{J}_{\mathcal{W} b}$ is that the external work exerted on the incoming flow on the boundary is already included in $\mathcal{P}_{\text {ext }}^{\text {total }}$.

Remark 10 - When restricting the model to the case of small perturbations, a straightforward linearized form of (60) can be considered leading to equations equivalent to the Biot system [7, 49], which has been mathematically studied using Galerkin approximations [43] or semigroup theory [23] in the light of an energy balance similar to (66). More precisely, in order to recover the Biot system from (60) we just need to remark that, in the linearized case for 
an incompressible fluid, $p$ and $m$ are linearly linked to the skeleton deformation tensor trace. Indeed, we get from (52) after linearization

$$
p=-K\left(\operatorname{tr} \underline{\underline{\varepsilon}}(\underline{y})-\frac{m}{\rho_{\mathrm{f}}}\right),
$$

with $K$ a positive constant involving the skeleton bulk modulus. Thus we can rewrite the mass conservation as

$$
\frac{1}{K} \frac{\mathrm{d} p}{\mathrm{dt}}+\underline{\nabla} \cdot \underline{v}_{\mathrm{s}}+\underline{\nabla} \cdot\left(\phi\left(\underline{v}_{\mathrm{f}}-\underline{v}_{\mathrm{s}}\right)\right)=\theta,
$$

similar to the classical form of the mass conservation in the Biot system.

Note finally that, even in large displacement cases, when existence results exist they usually make use of energy estimate like (66), see for instance [10] for comparable systems.

\section{Example of conservative time discretization}

We already emphasized in Remark 10 how the mathematical analysis can use the energy balance as an essential ingredient. The purpose of this section is to illustrate now the benefits of a complete energy balance for the system in the derivation of a stable discretization. The main idea is essentially to start from known conservative time schemes for both the solid and the fluid taken individually, and then to mimic at the discrete level the specific fluid-structure interaction terms, so that the discretization follows discrete conservation rules from one time step to the next.

\subsection{Time discretization scheme}

The scheme we propose here is the combination of:

- a mid-point energy-conserving extension of the mid-point Newmark scheme for the main mechanical equations, namely, with a specific treatment of the passive stress tensor according to [27] (see also [28]);

- a second-order Crank-Nicholson scheme inspired from [36] with specific care in the discretization of the Darcy term to respect the energy balance in the fluid-skeleton interaction.

We will thus propose a time-discrete version of System (63) expressing the equilibrium at time $t^{n+\frac{1}{2}}=\frac{1}{2}\left(t^{n}+t^{n+1}\right)$ as an algorithm for inferring the solution at time $t^{n+1}$ from that at $t^{n}$. In order to enforce the energy-wise stability of this procedure, the key point lies in adequate definitions of the quantities needed at time $t^{n+\frac{1}{2}}$. In the sequel we use the standard notation

$$
g^{n+\frac{1}{2}}=\frac{g^{n}+g^{n+1}}{2}
$$

except when otherwise specified for some variables that we denote by $g^{n+\frac{1}{2} \sharp}$. For example, recalling the definitions $m_{\mathrm{f}}=\rho_{\mathrm{f}} J \phi$ and $\underline{k}_{\mathrm{f}}=\sqrt{m_{\mathrm{f}}} \underline{\mathrm{f}}_{\mathrm{f}}$, we introduce the discretization

$$
\underline{k}_{\mathrm{f}}^{n+\frac{1}{2}}=\frac{\underline{k}_{\mathrm{f}}^{n+1}+\underline{k}_{\mathrm{f}}^{n}}{2}, \quad m_{\mathrm{f}}^{n+\frac{1}{2}}=\frac{m_{\mathrm{f}}^{n+1}+m_{\mathrm{f}}^{n}}{2},
$$


and we define

$$
\begin{aligned}
\left.\frac{\partial \underline{k}_{\mathrm{f}}}{\partial t}\right|_{\underline{\xi}} ^{n+\frac{1}{2} \sharp} & =\frac{\underline{k}_{\mathrm{f}}^{n+1}-\underline{k}_{\mathrm{f}}^{n}}{\Delta t} \\
\underline{v}_{\mathrm{f}}^{n+\frac{1}{2} \sharp} & =\frac{\underline{k}_{\mathrm{f}}^{n+\frac{1}{2}}}{\sqrt{m_{\mathrm{f}}} n+\frac{1}{2}}
\end{aligned}
$$

and

$$
\phi^{n+\frac{1}{2} \sharp}=\frac{m_{\mathrm{f}}^{n+\frac{1}{2}}}{\rho_{\mathrm{f}} J\left(\underline{y}_{\mathrm{s}}^{n+\frac{1}{2}}\right)}, \quad m^{n+\frac{1}{2}}=m_{\mathrm{f}}^{n+\frac{1}{2}}-\rho_{\mathrm{f}} \phi_{0} .
$$

For the solid, the non-standard mid-point variables rely on classical rules for the strain values

$$
\begin{gathered}
\underline{\underline{e}}^{n+\frac{1}{2} \sharp}=\underline{\underline{e}}\left(\underline{y}^{n+\frac{1}{2}}\right), \\
\mathrm{d}_{\underline{y}} \underline{e}^{n+\frac{1}{2} \sharp} \cdot \underline{v}^{*}=\frac{1}{2}\left(\underline{\underline{F}}\left(\underline{y}^{n+\frac{1}{2}}\right)^{T} \cdot \underline{\underline{\nabla}}_{\underline{\xi}} \underline{v}^{*}+\underline{\nabla}_{\underline{\xi}}^{T} \underline{v}^{*} \cdot \underline{\underline{F}}\left(\underline{y}^{n+\frac{1}{2}}\right)\right), \\
\underline{\underline{e}}^{n+\frac{1}{2} \sharp}=\frac{\underline{\underline{e}}^{n+1}-\underline{\underline{e}}^{n}}{\Delta t},
\end{gathered}
$$

and we recall the usual mid-point Newmark identity

$$
\underline{v}^{n+\frac{1}{2}}=\frac{\underline{v}^{n+1}+\underline{v}^{n}}{2}=\frac{\underline{y}^{n+1}-\underline{y}^{n}}{\Delta t} .
$$

We then discretize System (63) as follows

$$
\left\{\begin{array}{c}
\int_{\Omega_{0}} \rho_{\mathrm{s} 0}\left(1-\phi_{0}\right) \frac{\underline{v}_{\mathrm{s}}^{n+1}-\underline{v}_{\mathrm{s}}^{n}}{\Delta t} \cdot \underline{v}^{*} d \Omega+\mathcal{P}_{i}^{\mathrm{f}, n+\frac{1}{2} \sharp}\left(\underline{v}^{*}\right)+\int_{\Omega_{0}} \underline{\underline{\Sigma}}^{n+\frac{1}{2} \sharp}: d_{\underline{y}} \underline{e}^{n+\frac{1}{2} \sharp} \cdot \underline{v}^{*} d \Omega \\
=\int_{\Omega_{0}} \rho_{0} \underline{f}^{n+\frac{1}{2}} \cdot \underline{v}^{*} d \Omega+\int_{\partial \Omega_{0}} \underline{t}_{0}^{n+\frac{1}{2}} \cdot \underline{v}^{*} d S, \\
\mathcal{P}_{i}^{\mathrm{f}, n+\frac{1}{2} \sharp}\left(\underline{v}^{*}\right)+\int_{\Omega_{n+\frac{1}{2}}}\left(\phi^{n+\frac{1}{2} \sharp}\right)^{2}\left(\underline{v}_{\mathrm{f}}^{n+\frac{1}{2} \sharp}-\underline{v}_{\mathrm{s}}^{n+\frac{1}{2}}\right) \cdot \underline{D}_{\mathrm{f}} \cdot \underline{v}^{*} d \Omega \\
=\int_{\Omega_{n+\frac{1}{2}}}\left(-\phi^{n+\frac{1}{2} \sharp} \underline{\nabla}_{\underline{\mathrm{x}}} p^{n+\frac{1}{2}}+\phi^{n+\frac{1}{2} \sharp} \underline{f}^{n+\frac{1}{2}}\right) \cdot \underline{v}^{*} d \Omega \\
\int_{\Omega_{0}} q^{*} \frac{m_{\mathrm{f}}^{n+1}-m_{\mathrm{f}}^{n}}{\Delta t} d \Omega+\int_{\Omega_{n+\frac{1}{2}}} \underline{\nabla}_{\underline{\mathrm{x}}} \cdot\left(\rho_{\mathrm{f}} \phi^{n+\frac{1}{2} \sharp}\left(\underline{v}_{\mathrm{f}}^{n+\frac{1}{2} \sharp}-\underline{v}_{\mathrm{s}}^{n+\frac{1}{2}}\right)\right) q^{*} d \Omega=\int_{\Omega_{n+\frac{1}{2}}} q^{*} \theta^{n+\frac{1}{2}} d \Omega
\end{array}\right.
$$

to which we add the boundary conditions

$$
\underline{v}_{\mathrm{f}}^{n+\frac{1}{2} \sharp}=\underline{v}_{\mathrm{s}}^{n+\frac{1}{2}} \text { on } \partial \Omega_{n+\frac{1}{2}} .
$$

In System (70) we make use of the following discretization choices

$$
\begin{aligned}
\mathcal{P}_{i}^{\mathrm{f}, n+\frac{1}{2} \sharp}\left(\underline{v}^{*}\right) & =\int_{\Omega_{0}} \frac{\underline{k}_{\mathrm{f}}^{n+1}-\underline{k}_{\mathrm{f}}^{n}}{\Delta t} \cdot{\sqrt{m_{\mathrm{f}}}}^{n+\frac{1}{2}} \underline{v}^{*} d \Omega-\frac{1}{2} \int_{\Omega_{n+\frac{1}{2}}} \underline{\nabla}_{\underline{\mathrm{x}}} \cdot\left(\rho_{\mathrm{f}} \phi\left(\underline{v}_{\mathrm{f}}^{n+\frac{1}{2} \sharp}-v_{\mathrm{s}}^{n+\frac{1}{2}}\right)\right) \underline{v}_{\mathrm{f}}^{n+\frac{1}{2} \sharp} \cdot \underline{v}^{*} d \Omega \\
& +\int_{\Omega_{n+\frac{1}{2}}} \underline{\nabla}_{\underline{\mathrm{x}}} \cdot\left(\rho_{\mathrm{f}} \phi^{n+\frac{1}{2} \sharp} \underline{v}_{\mathrm{f}}^{n+\frac{1}{2} \sharp} \otimes\left(\underline{v}_{\mathrm{f}}^{n+\frac{1}{2} \sharp}-v_{\mathrm{s}}^{n+\frac{1}{2}}\right)\right) \cdot \underline{v}^{*} d \Omega-\frac{1}{2} \int_{\Omega_{n+\frac{1}{2}}} \theta \underline{v}_{\mathrm{f}}^{n+\frac{1}{2} \sharp} \cdot \underline{v}^{*} d \Omega . \quad(71)
\end{aligned}
$$


Regarding the second Piola-Kirchhoff stress tensor we draw our inspiration from $[28,27]$ and propose

$$
\underline{\underline{\underline{\Sigma}}}^{n+\frac{1}{2} \sharp}=\left.\frac{\partial \Psi}{\partial \underline{\underline{e}}}\right|^{n+\frac{1}{2} \sharp}+\left.\frac{\partial \Psi_{\mathrm{damp}}}{\partial \underline{\underline{\dot{e}}}}\right|^{n+\frac{1}{2} \sharp},
$$

with the viscous contribution given by

$$
\left.\frac{\partial \Psi_{\text {damp }}}{\partial \underline{\underline{\dot{e}}}}\right|^{n+\frac{1}{2} \sharp}=\frac{\partial \Psi_{\text {damp }}}{\partial \underline{\underline{\dot{e}}}}\left(\underline{\underline{e}}^{n+\frac{1}{2} \sharp}, \underline{\underline{e}}^{n+\frac{1}{2} \sharp}\right),
$$

and the hyperelastic part by

$$
\begin{aligned}
\left.\frac{\partial \Psi}{\partial \underline{\underline{e}}}\right|^{n+\frac{1}{2} \sharp} & =\frac{\partial \Psi}{\partial \underline{\underline{e}}}\left(\underline{\underline{e}}^{n+\frac{1}{2} \sharp}, m^{n+\frac{1}{2}}\right) \\
& +\left(\frac{\Psi\left(\underline{\underline{e}}^{n+1}, m^{n+\frac{1}{2}}\right)-\Psi\left(\underline{\underline{e}}^{n}, m^{n+\frac{1}{2}}\right)}{\Delta t}-\frac{\partial \Psi}{\partial \underline{\underline{e}}}\left(\underline{\underline{e}}^{n+\frac{1}{2} \sharp}, m^{n+\frac{1}{2}}\right): \underline{\underline{e}}^{n+\frac{1}{2}}\right) \frac{\underline{\underline{e}}^{n+\frac{1}{2}}}{\underline{\underline{e}}^{n+\frac{1}{2}}: \underline{\underline{e}}^{n+\frac{1}{2}}},
\end{aligned}
$$

while the pressure is obtained by

$$
\frac{p^{n+\frac{1}{2}}}{\rho_{\mathrm{f}}}=\left.\frac{\partial \Psi}{\partial m}\right|^{n+\frac{1}{2} \sharp}=\frac{\Psi\left(\underline{\underline{e}}^{n+1}, m^{n+1}\right)-\Psi\left(\underline{\underline{e}}^{n+1}, m^{n+\frac{1}{2}}\right)+\Psi\left(\underline{\underline{e}}^{n}, m^{n+\frac{1}{2}}\right)-\Psi\left(\underline{\underline{e}}^{n}, m^{n}\right)}{m^{n+1}-m^{n}} .
$$

We point out that the above two discrete expressions for the derivatives of the free energy have well-defined limits when $\left(\underline{\underline{e}}^{n+1}-\underline{\underline{e}}^{n}\right)$ and $\left(m^{n+1}-m^{n}\right)$ tend to zero, respectively, viz.

$$
\left.\frac{\partial \Psi}{\partial \underline{\underline{e}}}\right|^{n+\frac{1}{2} \sharp} \rightarrow \frac{\partial \Psi}{\partial \underline{\underline{e}}}\left(\underline{\underline{e}}^{n+\frac{1}{2} \sharp}, m^{n+\frac{1}{2}}\right),\left.\quad \frac{\partial \Psi}{\partial m}\right|^{n+\frac{1}{2} \sharp} \rightarrow \frac{1}{2}\left(\frac{\partial \Psi}{\partial m}\left(\underline{\underline{e}}^{n+1}, m^{n+\frac{1}{2}}\right)+\frac{\partial \Psi}{\partial m}\left(\underline{\underline{e}}^{n}, m^{n+\frac{1}{2}}\right)\right),
$$

and these limits should be used whenever the denominators vanish in the expressions (74) and (75).

\subsubsection{Stability analysis}

We can now establish the consistency and stability of the proposed time scheme, as the above discretization choices allow to reproduce the manipulations used in the time-continuous conservation. The result is summarized in the following theorem.

\section{Theorem 8}

The time scheme (70) is unconditionally stable, and of second-order accuracy.

Proof. Second-order accuracy is straightforward, since all discretized variables follow mid-point rules. For the stability analysis, we consider zero external forces and fluid sources, and we then aim at proving that the total discrete energy rate

$$
\frac{\mathcal{K}^{n+1}-\mathcal{K}^{n}}{\Delta t}+\frac{\Psi^{n+1}-\Psi^{n}}{\Delta t}
$$

decreases due to dissipative effects. To that purpose, we follow a strategy directly inspired from the proof of Theorem 7 by testing the - here discrete - variational formulation by similar quantities. We thus first analyze the fluid inertia power applied to the mid-point fluid velocity. We start with the same identities as in the time-continuous case to get

$$
\begin{aligned}
\mathcal{P}_{i}^{\mathrm{f}, n+\frac{1}{2} \sharp}\left(\underline{v}_{\mathrm{f}}^{n+\frac{1}{2} \sharp}\right)=\int_{\Omega_{0}} \frac{\underline{k}_{\mathrm{f}}^{n+1}-\underline{k}_{\mathrm{f}}^{n}}{\Delta t} \cdot{\sqrt{m_{\mathrm{f}}}}^{n+\frac{1}{2}} \underline{v}_{\mathrm{f}}^{n+\frac{1}{2} \sharp} d \Omega \\
+\int_{\Omega_{n+\frac{1}{2}}} \underline{\nabla}_{\underline{\mathrm{x}}} \cdot\left(\frac{1}{2} \rho_{\mathrm{f}} \phi^{n+\frac{1}{2} \sharp}\left(\underline{v}_{\mathrm{f}}^{n+\frac{1}{2} \sharp}\right)^{2}\left(\underline{v}_{\mathrm{f}}^{n+\frac{1}{2} \sharp}-\underline{v}_{\mathrm{s}}^{n+\frac{1}{2}}\right)\right) d \Omega
\end{aligned}
$$


Using the property that $\underline{v}_{\mathrm{f}}^{n+\frac{1}{2} \sharp}=\underline{v}_{\mathrm{s}}^{n+\frac{1}{2}}$ on $\partial \Omega$, we obtain

$$
\mathcal{P}_{i}^{\mathrm{f}, n+\frac{1}{2} \sharp}\left(\underline{v}_{\mathrm{f}}^{n+\frac{1}{2} \sharp}\right)=\int_{\Omega_{0}} \frac{\left(\underline{k}_{\mathrm{f}}^{n+1}\right)^{2}-\left(\underline{k}_{\mathrm{f}}^{n}\right)^{2}}{2 \Delta t} d \Omega=\frac{\mathcal{K}_{\mathrm{f}}^{n+1}-\mathcal{K}_{\mathrm{f}}^{n}}{\Delta t} .
$$

Adding the solid weak form $(70)_{1}$ applied to $\underline{v}_{\mathrm{s}}^{n+\frac{1}{2}}$ and the fluid weak form $(70)_{2}$ applied to $\left(\underline{v}_{\mathrm{f}}^{n+\frac{1}{2} \sharp}-\underline{v}_{\mathrm{s}}^{n+\frac{1}{2}}\right)$ we obtain

$$
\begin{gathered}
\frac{\mathcal{K}_{\mathrm{s}}^{n+1}-\mathcal{K}_{\mathrm{s}}^{n}}{\Delta t}+\frac{\mathcal{K}_{\mathrm{f}}^{n+1}-\mathcal{K}_{\mathrm{f}}^{n}}{\Delta t}+\left.\int_{\Omega_{0}} \frac{\partial \Psi}{\partial \underline{\underline{e}}}\right|^{n+\frac{1}{2} \sharp}: d_{\underline{y}} \underline{\underline{e}}^{n+\frac{1}{2} \sharp} \cdot \underline{v}_{\mathrm{s}}^{n+\frac{1}{2}} d \Omega \\
+\int_{\Omega_{n+\frac{1}{2}}}-\phi^{n+\frac{1}{2} \sharp} \underline{\nabla}_{\underline{\mathrm{x}}} p^{n+\frac{1}{2}} \cdot\left(\underline{v}_{\mathrm{f}}^{n+\frac{1}{2} \sharp}-\underline{v}_{\mathrm{s}}^{n+\frac{1}{2}}\right) d \Omega=-\left.\int_{\Omega_{0}} \frac{\partial \Psi_{\mathrm{damp}}}{\partial \underline{\underline{\dot{e}}}}\right|^{n+\frac{1}{2} \sharp}: d_{\underline{y}} \underline{\underline{e}}^{n+\frac{1}{2} \sharp} \cdot \underline{v}_{\mathrm{s}}^{n+\frac{1}{2}} d \Omega \\
\quad-\int_{\Omega_{n+\frac{1}{2}}}\left(\phi^{n+\frac{1}{2} \sharp}\right)^{2}\left(\underline{v}_{\mathrm{f}}^{n+\frac{1}{2} \sharp}-\underline{v}_{\mathrm{s}}^{n+\frac{1}{2}}\right) \cdot \underline{D}_{\mathrm{f}} \cdot\left(\underline{v}_{\mathrm{f}}^{n+\frac{1}{2} \sharp}-\underline{v}_{\mathrm{s}}^{n+\frac{1}{2}}\right) d \Omega .
\end{gathered}
$$

We convert the last term in the left hand side by using $(70)_{3}$ applied to $\frac{p^{n+\frac{1}{2}}}{\rho_{\mathrm{f}}}$,

$$
\begin{aligned}
\int_{\Omega_{n+\frac{1}{2}}}-\phi^{n+\frac{1}{2} \sharp} \underline{\nabla}_{\underline{x}} p^{n+\frac{1}{2}} \cdot\left(\underline{v}_{\mathrm{f}}^{n+\frac{1}{2} \sharp}-\underline{v}_{\mathrm{s}}^{n+\frac{1}{2}}\right) d \Omega & =\int_{\Omega_{0}} \frac{p^{n+\frac{1}{2}}}{\rho_{\mathrm{f}}} \frac{m_{\mathrm{f}}^{n+1}-m_{\mathrm{f}}^{n}}{\Delta t} d \Omega \\
& =\left.\int_{\Omega_{0}} \frac{\partial \Psi}{\partial m}\right|^{n+\frac{1}{2} \sharp} \frac{m^{n+1}-m^{n}}{\Delta t} d \Omega .
\end{aligned}
$$

Furthermore, using the classical identity (based on the linearity of $\underline{\underline{F}}$ )

$$
\begin{aligned}
\mathrm{d}_{\underline{y}} \underline{\underline{e}}^{n+\frac{1}{2} \sharp} \cdot \underline{v}_{\mathrm{s}}^{n+\frac{1}{2}} & =\frac{1}{2}\left(\underline{\underline{F}}\left(\underline{\underline{v}}_{\mathrm{s}}^{n+\frac{1}{2}}\right)^{T} \cdot \mathrm{d}_{\underline{y}} \underline{\underline{F}} \cdot \underline{v}_{\mathrm{s}}^{n+\frac{1}{2}}+\left(\mathrm{d} \underline{\underline{\underline{F}}} \cdot \underline{\underline{v}}_{\mathrm{s}}^{n+\frac{1}{2}}\right)^{T} \cdot \underline{\underline{F}}\left(\underline{\underline{y}}^{n+\frac{1}{2}}\right)\right) \\
& =\frac{1}{2}\left(\left(\frac{\underline{\underline{F}}^{n+1}+\underline{\underline{F}}^{n}}{2}\right)^{T} \cdot \frac{\underline{\underline{F}}^{n+1}-\underline{\underline{F}}^{n}}{\Delta t}+\left(\frac{\underline{\underline{F}}^{n+1}-\underline{\underline{F}}^{n}}{\Delta t}\right)^{T} \cdot \frac{\underline{F}^{n+1}+\underline{\underline{F}}^{n}}{2}\right) \\
& =\frac{1}{\Delta t}\left(\left(\underline{\underline{F}}^{n+1}\right)^{T} \cdot \underline{\underline{F}}^{n+1}-\left(\underline{\underline{F}}^{n}\right)^{T} \cdot \underline{\underline{F}}^{n}\right)=\frac{\underline{\underline{e}}^{n+1}-\underline{\underline{e}}^{n}}{\Delta t},
\end{aligned}
$$

we obtain

$$
\begin{aligned}
\left.\frac{\partial \Psi}{\partial \underline{\underline{e}}}\right|^{n+\frac{1}{2} \sharp}: d_{\underline{y}} \underline{\underline{e}} \underline{\underline{e}}^{n+\frac{1}{2} \sharp} \cdot \underline{v}_{\mathrm{s}}^{n+\frac{1}{2}} & +\left.\frac{\partial \Psi}{\partial m}\right|^{n+\frac{1}{2} \sharp} \frac{m^{n+1}-m^{n}}{\Delta t} \\
& =\left.\frac{\partial \Psi}{\partial \underline{\underline{e}}}\right|^{n+\frac{1}{2} \sharp}: \frac{\underline{\underline{e}}^{n+1}-\underline{\underline{e}}^{n}}{\Delta t}+\left.\frac{\partial \Psi}{\partial m}\right|^{n+\frac{1}{2} \sharp} \frac{m^{n+1}-m^{n}}{\Delta t} \\
& =\frac{\Psi^{n+1}-\Psi^{n}}{\Delta t},
\end{aligned}
$$

as directly follows (by construction, indeed) from (74) and (75). Finally, we obtain

$$
\begin{aligned}
\frac{\mathcal{K}^{n+1}-\mathcal{K}^{n}}{\Delta t}+\frac{\Psi^{n+1}-\Psi^{n}}{\Delta t}=- & \int_{\Omega_{0}} \frac{\partial \Psi_{\text {damp }}}{\partial \underline{\underline{\dot{e}}}}\left(\underline{\underline{e}}^{n+\frac{1}{2} \sharp}, \underline{\underline{e}}^{n+\frac{1}{2} \sharp}\right): \underline{\underline{e}}^{n+\frac{1}{2}} d \Omega \\
& -\int_{\Omega_{n+\frac{1}{2}}}\left(\phi^{n+\frac{1}{2} \sharp}\right)^{2}\left(\underline{v}_{\mathrm{f}}^{n+\frac{1}{2} \sharp}-\underline{v}_{\mathrm{s}}^{n+\frac{1}{2}}\right) \cdot \underline{\underline{D}}_{\mathrm{f}} \cdot\left(\underline{v}_{\mathrm{f}}^{n+\frac{1}{2} \sharp}-\underline{v}_{\mathrm{s}}^{n+\frac{1}{2}}\right) d \Omega \leq 0 .
\end{aligned}
$$

which is the discrete counterpart of (66), showing that the proposed time scheme unconditionally preserves the physical energy balance of the system. 


\section{Concluding remarks}

We have proposed a complete framework for modeling finite strain poromechanics in a very general setting, the only specific assumptions made being fluid incompressibility and isothermal conditions. Our construction entirely relies on the crucial conservation and thermodynamics principles, and as a consequence we very naturally established a global energy balance for the resulting coupled system, directly from the weak formulation.

This fundamental energy balance is very important for further mathematical analyses of the system. Moreover, it also provides a milestone for discretizing the formulation, as fulfilling similar balances for the discretized solution is a key step in the stability analysis, as illustrated in Section 7. This type of approach is for example already very effectively used in the numerical analysis of fluid-structure interaction problems - see e.g. [36] - to which we showed that our proposed formulation bears some resemblance. In this respect, adequate time discretization strategies - e.g. implementing the coupling conditions in alternative forms compared to the monolithic scheme proposed in Section 7 - are a natural perspective for our work. Note that spatial discretization issues also remain to be addressed, in particular when considering incompressibility in the solid phase, in order to circumvent numerical locking phenomena [4], see also

[2] for various numerical aspects concerning this type of coupled problem.

Finally, we emphasize that the proposed formulation leaves much room for modeling very general constitutive behaviors. This holds both for the fluid component - within the incompressibility assumption - for which we can consider e.g. non-Newtonian stress laws and various forms of permeability modeling, and also for the skeleton which is modeled based on a generalized hyperelastic potential, with optional additional dissipative effects. The hyperelastic potential allows to represent a wide range of mechanical behaviors - both compressible and incompressible, finite strain conditions, arbitrary anisotropy in particular - and with some extensions could also include some plasticity effects and active components, the latter based e.g. on coupling with chemical effects [22]. We expect this wide range of applicability to be of utmost value in many fields, including - but not restricted to - soft tissue biomechanics which originally motivated this work.

\section{References}

[1] E.S. Almeida and R.L. Spilker. Finite element formulations for hyperelastic transversely isotropic biphasic soft tissues. Computer Methods in Applied Mechanics and Engineering, 151(3-4):513-538, 1998.

[2] S. Badia, A. Quaini, and A. Quarteroni. Coupling Biot and Navier-Stokes equations for modelling fluid-poroelastic media interaction. J. Comput. Phys., 228(21):7986-8014, 2009.

[3] L. Baffico, C. Grandmont, and B. Maury. Multiscale modelling of the respiratory tract. Mathematical Models and Methods in Applied Sciences (M3AS), 20(1):59-93, 2010.

[4] K.J. Bathe. Finite Element Procedures. K.J. Bathe, 2007.

[5] M.A. Biot. General theory of three-dimensional consolidation. J. Appl. Phys., 12(155):155$164,1941$.

[6] M.A. Biot. Mechanics of deformation and acoustic propagation in porous media. J. Appl. Phys., 33(4):1482-1498, 1962.

[7] M.A. Biot. Theory of finite deformations of porous solids. Indiana University Mathematics Journal, 21(7):597-620, 1972. 
[8] J. Bluhm. Constitutive relations for thermo-elastic porous solids within the framework of finite deformations. IUTAM Symposium on Theoretical and Numerical Methods in Continuum Mechanics of Porous Materials, 87:27-32, 2001.

[9] R.I. Borja. On the mechanical energy and effective stress in saturated and unsaturated porous continua. International Journal of Solids and Structures, 43(6):1764-1786, 2006.

[10] M. Boulakia. Existence of weak solutions for an interaction problem between an elastic structure and a compressible viscous fluid. Journal de Mathématiques Pures et Appliquées, 84(11):1515-1554, 2005.

[11] R.M. Bowen. Incompressible porous media models by use of the theory of mixtures. Int. J. Engng Sci., 18:1129-1148, 1980.

[12] R.M. Bowen. Compressible porous media models by use of the theory of mixtures. Int. J. Engng Sci., 20(6):697-735, 1982.

[13] D. Chapelle, J.-F. Gerbeau, J. Sainte-Marie, and I.E. Vignon-Clementel. A poroelastic model valid in large strains with applications to perfusion in cardiac modeling. Computational Mechanics, 46(1):91-101, 2010.

[14] D. Chapelle, P. Le Tallec, P. Moireau, and M. Sorine. An energy-preserving muscle tissue model: formulation and compatible discretizations. Int. J. Multiscale Comput. Eng., 10(2):189-211, 2012.

[15] P. G. Ciarlet. Mathematical Elasticity, Vol. I : Three-Dimensional Elasticity. Studies in Mathematics and its Applications. North Holland, 1988.

[16] O. Coussy. Poromechanics. John Wiley and Sons, 2004.

[17] R. de Boer. Trends in Continuum Mechanics of Porous Media. Springer, 2005.

[18] E. de Langre. Effects of wind on plants. Annual Review of Fluid Mechanics, 40:141-168, 2008.

[19] S. Diebels and W. Ehlers. Dynamic analysis of a fully saturated porous medium accounting for geometrical and material non-linearities. International Journal for Numerical Methods in Engineering, 39(1):81-97, 1996.

[20] J. Donea, S. Giuliani, and J.P. Halleux. An arbitrary Lagrangian-Eulerian finite element method for transient dynamic fluid-structure interactions. Comp. Meth. Appl. Mech. Engng., 33(1-3):689-723, 1982.

[21] L. Dormieux, P. Barboux, O. Coussy, and P. Dangla. A macroscopic model of the swelling phenomenon of a saturated clay. European Journal of Mechanics A-Solids, 14(6):981-1004, 1995 .

[22] A.C. Eringen and J.D. Ingram. A continuum theory of chemically reacting media - I. Int. J. Eng. Sci., 3(2):197-212, 1965.

[23] A. Ezziani. Ondes dans les milieux poroélastiques, analyse du modèle de Biot. Revue Africaine de la Recherche en Informatique et Mathématiques Appliquées (ARIMA), 5:95109, 2006. 
[24] M.A. Fernández and J.-F. Gerbeau. Algorithms for fluid-structure interaction problems. In Cardiovascular Mathematics, volume 1 of MS\&A. Model. Simul. Appl., pages 307-346. Springer, 2009.

[25] Y.C. Fung. Biomechanics: Mechanical Properties of Living Tissues. Springer-Verlag, 2nd edition, 1993.

[26] A. Gajo. A general approach to isothermal hyperelastic modelling of saturated porous media at finite strains with compressible solid constituents. Proceedings of the Royal Society A-Mathematical Physical And Engineering Sciences, 2010. Published online.

[27] O. Gonzales. Exact energy and momentum conserving algorithm for general models in nonlinear elasticity. Comput. Meth. Appl. Mech. Eng., 190(13-14):1763-1783, 2000.

[28] P. Hauret and P. Le Tallec. Energy controlling time integration methods for nonlinear elastodynamics and low velocity impact. Comput. Meth. Appl. Mech. Eng., 195:4890-4916, 2006 .

[29] G.A. Holzapfel and R.W. Ogden, editors. Mechanics of Biological Tissue. Springer, 2006.

[30] U. Hornung. Homogenization and Porous Media. Interdisciplinary Applied Mathematics. Springer, 1997.

[31] J.M. Huyghe, T. Arts, D.H. van Campen, and R.S. Reneman. Porous medium finite element model of the beating left ventricle. Am J Physiol Heart Circ Physiol, 262(4):H1256-H1267, 1992.

[32] J.M. Huyghe and D.H. van Campen. Finite deformation theory of hierarchically arranged porous solids - II Constitutive behaviour. Int. J. Eng. Sci., 33(13):1873-1886, 1995.

[33] K. Johnson, P. Sharma, and J. Oshinski. Coronary artery flow measurement using navigator echo-gated phase contrast magnetic resonance velocity mapping at $3.0 \mathrm{~T}$. Journal of Biomechanics, 41(3):595-602, 2008.

[34] F. Kuwahara, Y. Sano, J. Liu, and A. Nakayama. A porous media approach for bifurcating flow and mass transfer in a human lung. Journal of Heat Transfer, 131(10):101013, 2009.

[35] P. Le Tallec. Numerical methods for nonlinear three-dimensional elasticity. In P. G. Ciarlet and J.-L. Lions, editors, Handbook of Numerical Analysis, volume 3. Elsevier, 1994.

[36] P. Le Tallec and P. Hauret. Energy conservation in fluid structure interactions. In Numerical methods for scientific computing. Variational problems and applications, pages 94-107, 2003.

[37] C. Li, R.I. Borja, and R.A. Regueiro. Dynamics of porous media at finite strain. Computer Methods in Applied Mechanics and Engineering, 193(36-38):3837-3870, 2004.

[38] B. Loret and F.M.F. Simoes. A framework for deformation, generalized diffusion, mass transfer and growth in multi-species multi-phase biological tissues. European Journal of Mechanics A-Solids, 24(5):757-781, 2005.

[39] K. May-Newman and A.D. McCulloch. Homogenization modeling for the mechanics of perfused myocardium. Progress in Biophysics and Molecular Biology, 69(2-3):463-481, 1998. 
[40] M.P. Nash and P.J. Hunter. Computational mechanics of the heart - From tissue structure to ventricular function. Journal of Elasticity, 61(1-3):113-141, 2000.

[41] E. Rohan and R. Cimrman. Two-scale modeling of tissue perfusion problem using homogenization of dual porous media. International Journal for Multiscale Computational Engineering, 8(1):81-102, 2010.

[42] J. Sainte-Marie, D. Chapelle, R. Cimrman, and M. Sorine. Modeling and estimation of the cardiac electromechanical activity. Computers \& Structures, 84(28):1743-1759, 2006.

[43] J.E. Santos. Elastic wave propagation in fluid-saturated porous media. i. the existence and uniqueness theorems. RAIRO Modélisation Mathématique et Analyse Numérique, 20(1):113-128, 1986.

[44] J.J.J. Shi, K.R. Rajagopal, and A.S. Wineman. Applications of the theory of interacting continua to the diffusion of a fluid through a non-linear elastic media. Int. J. Engng Sci., 19:871-889, 1981.

[45] N.P. Smith and G.S. Kassab. Analysis of coronary blood flow interaction with myocardial mechanics based on anatomical models. Philosophical Transactions of the Royal Society of London Series A-Mathematical Physical and Engineering Sciences, 359(1783):1251-1262, 2001.

[46] H. Tang and Y.C. Fung. Fluid movement in a channel with permeable walls covered by porous media: A model of lung alveolar sheet. Journal of Applied Mechanics, 42(1):45-50, 1975 .

[47] K. Terzaghi. Theoretical Soil Mechanics. John Wiley and Sons, 1943.

[48] R. Wooding. Steady state free thermal convection of liquid in a saturated permeable medium. Journal of Fluid Mechanics, 2(3):273-285, 1957.

[49] O.C. Zienkiewicz and T. Shiomi. Dynamic behaviour of saturated porous media; the generalized Biot formulation and its numerical solution. International Journal for Numerical and Analytical Methods in Geomechanics, 8:71-96, 1984. 